\title{
Three-dimensional MHD simulations of the magnetosphere of Uranus
}

\author{
Gábor Tóth ${ }^{1}$ and Dániel Kovács \\ Department of Atomic Physics, Eötvös University, Budapest, Hungary
}

Kenneth C. Hansen and Tamas I. Gombosi

Center for Space Environment Modeling, University of Michigan, Ann Arbor, Michigan, USA

Received 27 January 2004; revised 27 July 2004; accepted 7 September 2004; published 10 November 2004.

[1] We have successfully simulated the magnetosphere of Uranus for the time period of the Voyager 2 flyby in January 1986. On the basis of the Voyager measurements, a selfconsistent numerical solution is obtained with the parallel block adaptive three-dimensional (3-D) MHD code BATS-R-US. The time-dependent simulation has been carried out with a new explicit-implicit time integration scheme. By comparing corotating steady state solutions and a fully time-dependent 3-D simulation with the Voyager data, we show that the magnetosphere of Uranus at the time of the flyby can be regarded as stationary relative to the frame corotating with the planet. We obtained excellent agreement with the observed magnetic field vector along the whole path of the flyby, which includes the near-Uranus offset dipole field as well as several current sheet crossings in the tail. The location of the bow shock and the magnetopause also agree to high accuracy. We are confident that our numerical solution is a good representation of the three-dimensional magnetosphere of Uranus during the flyby. The numerical solution shows a twisted magnetotail with field lines that are also stretched due to the flow of plasma in the magnetotail. INDEX TERMS: 2756 Magnetospheric Physics: Planetary magnetospheres (5443, 5737, 6030); 2753 Magnetospheric Physics: Numerical modeling; 2784 Magnetospheric Physics: Solar wind/magnetosphere interactions; 5737 Planetology: Fluid Planets: Magnetospheres (2756); 7843 Space Plasma Physics: Numerical simulation studies; KEYWORDS: Uranus, magnetospheres, numerical modeling, magnetospheric configuration and dynamics

Citation: Tóth, G., D. Kovács, K. C. Hansen, and T. I. Gombosi (2004), Three-dimensional MHD simulations of the magnetosphere of Uranus, J. Geophys. Res., 109, A11210, doi:10.1029/2004JA010406.

\section{Introduction}

[2] In January of 1986, Voyager 2 had a close encounter with Uranus, and the observations carried out during that several-day-long period give the main base of our knowledge of this planet. The most surprising result was the extraordinarily large angle between the rotation and magnetic dipole axes $\left(58.61^{\circ}\right)$. The magnetic dipole axis is displaced by $0.31 \mathrm{R}_{\mathrm{U}}$ along the rotation axis from the center of the planet and has a magnitude of $22,836 n T R_{U}^{3}$, where $R_{U}$ denotes the radius of Uranus. These numbers resulted from the analysis of the magnetometer data by Ness et al. [1986], who introduced the offset tilted dipole (OTD) model. Later, the Q3 model created by Connerney et al. [1987] gave more precise values for these data and determined the dipole and quadrupole components of the magnetic field.

[3] The structure of the magnetosphere of Uranus at the time of the encounter is shown in Figure 1. Thick and dashed

\footnotetext{
${ }^{1}$ Also at Center for Space Environment Modeling, University of Michigan, Ann Arbor, Michigan, USA.

Copyright 2004 by the American Geophysical Union. 0148-0227/04/2004JA010406
}

lines denote the bow shock and magnetopause, respectively. The rotation axis $(\mathbf{R})$ is tilted by approximately $8^{\circ}$ away from the direction toward the Sun and lies almost exactly in the plane of Figure 1. The magnetic dipole axis $(\mathbf{M})$ is rotating in the anticlockwise direction if one looks at it from the Sun, causing much of the magnetosphere to rigidly corotate.

[4] Although the Voyager data obtained in 1986 has been analyzed in several papers (for example, Behannon et al. [1987], Connerney et al. [1987], Lepping [1994], Bridge et al. [1986], and Ness et al. [1986]), no self-consistent numerical modeling of the magnetosphere of Uranus has been published in the past 17 years. This is not surprising because the numerical codes that can solve the MHD equations in three dimensions with the resolution required by magnetospheric simulations have become available only recently. The key features of our numerical code which make these simulations possible are adaptive mesh refinement, implicit time stepping techniques, and parallel computing.

[5] Numerically modeling the magnetosphere of Uranus is a demanding test of modern computational schemes due to the fact that we have to solve the time-dependent MHD equations in three dimensions in an extended domain for several days of real time. In order to validate our model, we compare with Voyager 2 data. The agreement between 


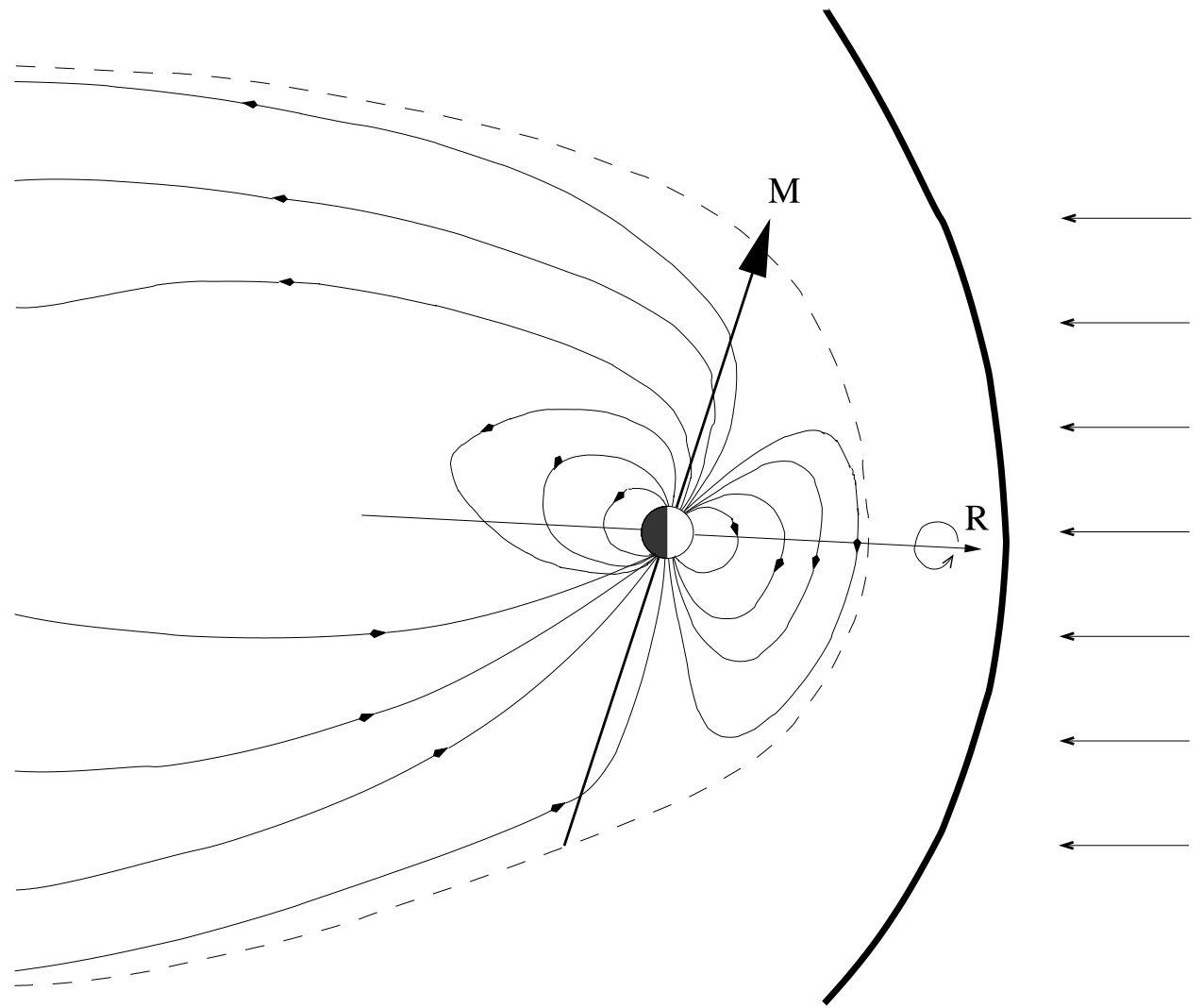

Figure 1. Schematic picture of the magnetosphere with the bow shock (thick line) and the magnetopause (dashed line) of Uranus. $\mathbf{M}$ and $\mathbf{R}$ denote the magnetic dipole and rotational axes, respectively. The rotational axis is tilted downward by approximately $8^{\circ}$ from the Sun-Uranus line. The arrows on the right represent the solar wind.

measurements and simulated results can confirm the accuracy of our physical assumptions as well as the accuracy of our numerical techniques. A successful comparison yields more than just a confirmation that we are solving the right equations with good numerical methods. In effect we transform a one-dimensional (1-D) data set measured along the path of Voyager into a full three-dimensional timedependent representation of the magnetosphere of Uranus. This transformation relies on our understanding of magnetospheric physics.

[6] The structure of this paper is as follows: first we describe the physical model, i.e., the ideal MHD equations both in inertial and rotating frames. Section 3 presents the code and the numerical techniques used in the simulations. Our gradual approach to the final time-dependent simulation is described in section 4 . The necessary transformations and processing of the measurements are detailed in section 5 . The numerical results are presented in section 6 . Finally, we close this work with a physical interpretation of the numerical model.

\section{Equations}

[7] We solve the ideal MHD equations in conservative form:

$$
\frac{\partial \rho}{\partial t}+\nabla \cdot(\rho \mathbf{u})=0
$$

$$
\begin{aligned}
\frac{\partial(\rho \mathbf{u})}{\partial t}+ & \nabla \cdot\left[\rho \mathbf{u} \mathbf{U}+\left(p+\frac{B^{2}}{2 \mu_{0}}\right) I-\frac{\mathbf{B B}}{\mu_{0}}\right] \\
& =\rho \mathbf{g}+\rho \Omega^{2} \mathbf{d}+2 \rho \mathbf{u} \times \mathbf{\Omega} \\
\frac{\partial \mathbf{b}}{\partial t}+\nabla \cdot(\mathbf{u B}-\mathbf{B u})=0 & \\
\frac{\partial \varepsilon}{\partial t}+\nabla \cdot\left[\mathbf{u}\left(\varepsilon+p+\frac{B^{2}}{2 \mu_{0}}\right)-\frac{\mathbf{u} \cdot(\mathbf{B B})}{\mu_{0}}\right] & =\rho \mathbf{g} \cdot \mathbf{u}+\rho \Omega^{2} \mathbf{d} \cdot \mathbf{u}
\end{aligned}
$$

where $I$ is identity matrix, $\rho$ denotes the mass density of the plasma, $\mathbf{u}$ is the plasma velocity vector, $\mathbf{B}$ is magnetic induction, $p$ is the pressure, $\mathbf{g}$ is the gravitational acceleration, $\mu_{0}$ is the magnetic permeability of vacuum, and $\gamma$ is the ratio of specific heats which we take to be $5 / 3$. When solving in a reference frame rotating with Uranus, terms appear in the momentum and energy equations that represent the Coriolis and centrifugal forces. These terms, characterized by an angular velocity vector $\Omega$ and a radial position vector $\mathbf{d}$ (orthogonal to the rotation axis), are also included.

[8] The total energy density $\varepsilon$ is the sum of the thermal, kinetic, and magnetic energy densities: 


$$
\varepsilon=\frac{p}{\gamma-1}+\frac{\rho u^{2}}{2}+\frac{B^{2}}{2 \mu_{0}}
$$

Solving the conservative system of equations with a conservative numerical scheme ensures that the jump conditions across shock waves, such as the bow shock, are correctly obtained. Inside the bow shock, however, we have the option of using the nonconservative pressure equation

$$
\frac{\partial p}{\partial t}+\nabla \cdot(p \mathbf{u})+(\gamma-1) p \nabla \cdot \mathbf{u}=0
$$

instead of equation (4) to avoid negative pressures that can arise from discretization errors in regions dominated by strong magnetic fields (i.e., with very low plasma-beta). In all simulations presented in this paper, the pressure equation (6) is used within $19 \mathrm{R}_{\mathrm{U}}$. Since the bow shock is outside this region, the proper jump conditions are obtained.

\section{BATS-R-US Numerical Scheme}

[9] The BATS-R-US (Block Adaptive Tree Solar-wind Roe Upwind Scheme) [Powell et al., 1999] is a shockcapturing numerical scheme that solves the time-dependent ideal MHD equations in 3-D. BATS-R-US is used mostly for simulating magnetospheres of planets and comets and for space environment modeling. It is being developed at the University of Michigan.

[10] BATS-R-US uses block-adaptive mesh refinement (AMR) to create the most suitable grid for a given problem according to the physical conditions and circumstances. The block-based AMR is well suited to parallel computers, since blocks can be distributed evenly among the processors. The grid resolution can be changed on either physical or geometrical basis. The equations are discretized spatially with total variation diminishing [Harten, 1983] MUSCL (Monotonic Upwind Scheme for Conservation Laws) type [van Leer, 1979] finite volume schemes. In the simulations presented in this paper the numerical flux is based on the second-order local artificial wind scheme by Sokolov et al. [2002]. The error in the divergence of the magnetic field is kept at the truncation error level with the eight-wave scheme [Powell, 1994].

[11] There are various options for time discretization. Steady state solutions can be efficiently obtained with local time stepping. In this case, the time step is set individually for every grid cell based on the local numerical stability conditions. Although the cells march toward steady state at different rates, the final steady state solution is the physically correct one due to the balance of fluxes and sources for all cells independent of the time step.

[12] In order to follow the time evolution of a system, local time stepping cannot be used because the same time step is required for every cell. There are two classes of time-accurate time stepping methods: explicit and implicit. In explicit time stepping schemes the time step is set by the cell with the most restrictive stability condition. The stability condition requires a wave to propagate a distance less than one cell length during a single time step. In magnetospheric simulations this can lead to very small time steps because the smallest cells are close to the planet where the magnetic field and hence the magnetosonic speeds are large. This restriction can be eliminated by the use of implicit time stepping schemes [Keppens et al., 1999; Tóth et al., 1998]. The time step in an implicit scheme is not limited by the wave speeds and therefore can be much larger than for an explicit scheme. However, the implicit scheme is more costly to compute. In BATS-R-US a highly parallel and efficient implicit numerical scheme has been implemented which uses Krylov subspace iterative solvers combined with Schwarz-type preconditioning. The preconditioning is done on a block by block basis. The efficiency is further improved by using explicit time stepping in the blocks which are stable for the given time step.

[13] For the time-accurate run presented in this paper, the explicit scheme would use $0.023 \mathrm{~s}$ time steps, while the implicit scheme can be run with $75 \mathrm{~s}$ time steps. Although the implicit scheme is a factor of 35 times more expensive per time step, it still runs about 90 times faster than the explicit time stepping thanks to the 3250 times larger times steps.

[14] In terms of accuracy the implicit and explicit schemes obtain essentially the same solution when the time variation is slow (quasi-steady state) because the implicit scheme uses the same "right-hand side" discretization as the explicit scheme. For a true steady state the explicit and implicit solutions coincide. For fast transients the explicit scheme is more accurate. In the simulations presented in this paper, the quasi-steady state assumption is fulfilled, since the rotation of the solution with a period of 17.24 hours is very slow relative to the $75 \mathrm{~s}$ time steps.

\section{Modeling Strategy}

[15] As it can be seen in Figure 1, during the Voyager flyby the rotational axis of Uranus was nearly aligned with the Uranus-Sun axis. If we neglect this small deviation and further assume that the upstream boundary conditions, i.e., the solar wind parameters, are cylindrically symmetric around the rotation axis, then it is plausible to assume that there is a corotating steady state solution. Obtaining such a steady state solution requires less effort than doing a full time-dependent simulation. In addition, it is generally easier to interpret a steady state solution than a time-dependent one. For these reasons, we present a corotating steady state model as well as a fully time-dependent model. The corotating steady state model solution serves as an initial condition and also as a reference solution for the timedependent calculations.

[16] We use the following three coordinate systems for the simulations. As illustrated in Figure 2, the USO (Uranus Solar Orbital) system is defined by the $X_{U S O}$ axis pointing toward the Sun, the $Y_{U S O}$ axis lying in the orbital plane and pointing opposite to the direction of motion of Uranus, and the $Z_{U S O}$ axis completes the right-handed system. The inertial USO system is used for time-dependent simulations.

[17] The USM (Uranus Solar Magnetic) frame rotates around the $X_{U S M}=X_{U S O}$ axis so that the USM and USO systems are aligned every 17.24 hours, which is one Uranian day. In the USM system the magnetic axis always lies in the $X_{U S M}-Z_{U S M}$ plane, and the $Y_{U S M}$ axis completes the right-handed system. The USM frame is therefore a rotating frame. If the tilt of the rotation axis relative to $X_{U S M}$ 


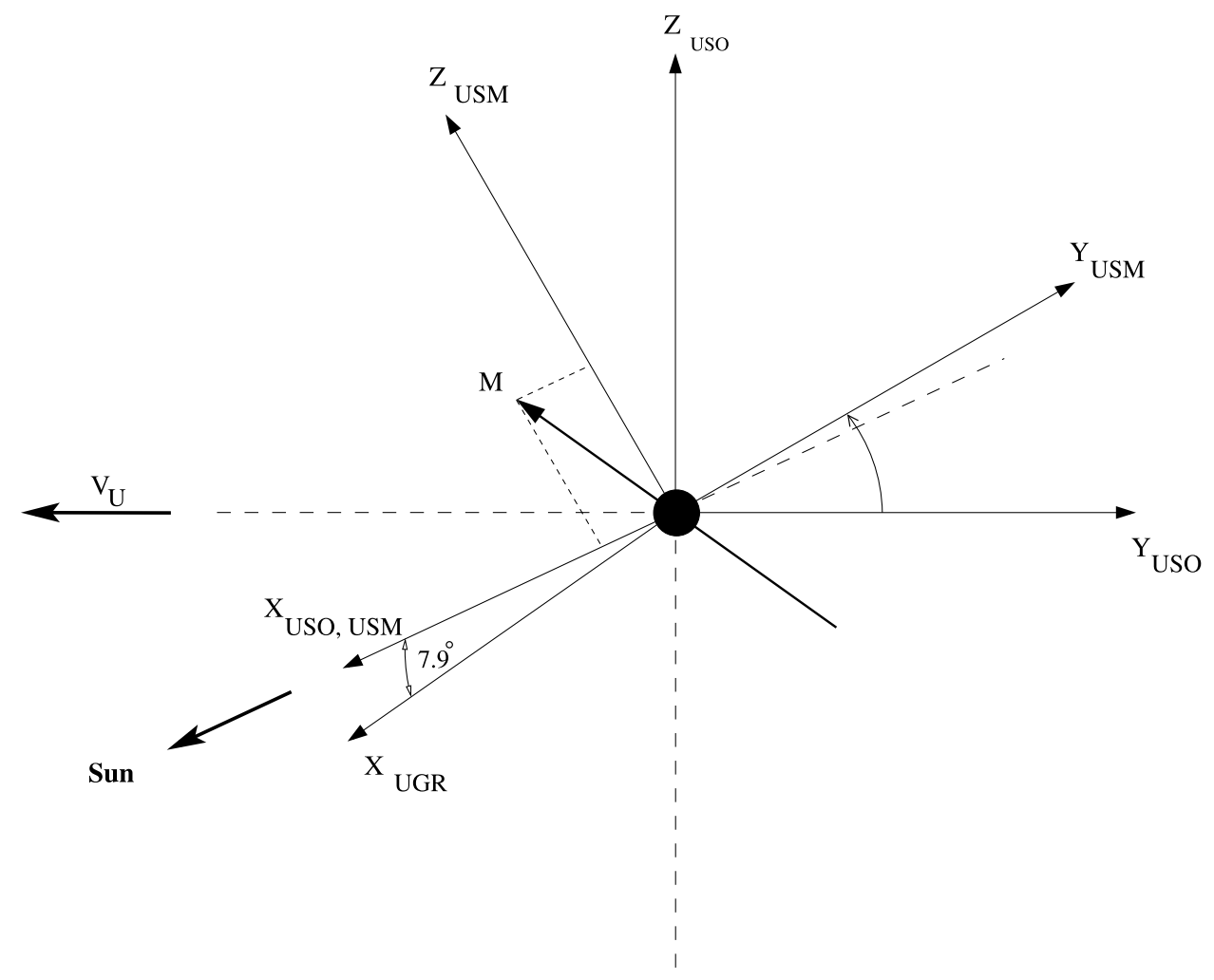

Figure 2. Coordinate systems used for modeling. The $X_{U S O, U S M}$ axis points toward the Sun and the $X_{U G R}$ axis makes an angle of $7.9^{\circ}$ to it. The $X_{U S O}-Y_{U S O}$ plane corresponds to the orbital plane and $V_{U}$ denotes the orbital velocity of Uranus. The magnetic axis $\mathbf{m}$ lies in the $X_{U S M}-Z_{U S M}$, as well as in the $X_{U G R}-Z_{U G R}$ planes.

is neglected, then the dipole is stationary in the corotating frame. We use this orientation for modeling the magnetosphere as a corotating steady state. Without this approximation, the rotation and the magnetic axes are not stationary in the USM system, they wobble back and forth by approximately $\pm 8^{\circ}$, and the angular velocity of the USM system is not exactly constant. Far from the planet, where the structure of the magnetosphere is mostly determined by the solar wind, the wobbling of the field near the planet is probably not very essential.

[18] The final coordinate system we define is the UGR (Uranographic) system. In this system the $X_{U G R}$ axis coincides with the real rotation axis that is tilted downward by $7.9^{\circ}$ relative to the $X_{U S O}=X_{U S M}$ axis. The UGR frame is fixed to the surface of the planet. For sake of convenience the $Z_{U G R}$ axis is defined such that the $X_{U G R}-Z_{U G R}$ plane contains the magnetic dipole. At the instance when the USM and USO frames coincide, the UGR system can be obtained from the USM/USO system by a $7.9^{\circ}$ rotation around the $Y_{U S O}$ axis. The UGR coordinate system is used to compare the measured and simulated data sets near the planet where the intrinsic magnetic field dominates over the effect of the solar wind.

[19] The computational domain extends from $-704 \mathrm{R}_{\mathrm{U}}$ to $+64 \mathrm{R}_{\mathrm{U}}$ in the $X$ direction and $-128 \mathrm{R}_{\mathrm{U}}$ to $+128 \mathrm{R}_{\mathrm{U}}$ in the $Y$ and $Z$ directions and the planet is centered at the origin; thus the boundaries are sufficiently far from the planet and there is no interference with the numerical solution along the Voyager trajectory. We wish to use the results of the corotating simulations as an initial condition for the time-dependent simulation. Because of this, it is useful to preserve the cylindrical symmetry of the grid as much as possible. For this reason, after some exploratory simulations, we designed a geometrically refined grid specifically adjusted for the Uranus magnetosphere. The block structure of this grid is depicted in Figure 18. The locations of the resolution changes are shown as black lines in the two cut planes. Each Cartesian block contains $4 \times 4 \times 4$ cells. In the various simulations the smallest cells near the planet are $1 / 8$ to $1 / 4 R_{U}$, while the largest cells far away are $8 \mathrm{R}_{\mathrm{U}}$. The computational domain is resolved with 5000 to 8000 blocks, which corresponds to 0.3 to 0.5 million grid cells.

[20] We impose supersonic inflow boundary conditions at $x=64 \mathrm{R}_{\mathrm{U}}$, which means that the solar wind values are set for all the MHD variables in the ghost cells. The boundary condition at $x=-704 \mathrm{R}_{\mathrm{U}}$ is simple outflow, which is well approximated with maintaining zero gradient in all flow variables. The other four edges of the computational domain at $y= \pm 128 \mathrm{R}_{\mathrm{U}}$ and $z= \pm 128 \mathrm{R}_{\mathrm{U}}$ are sufficiently far from the major features of the solution so that the flow hardly changes at these boundaries. Here the zero gradient boundary conditions were used for the simulations done in an inertial frame to minimize effects on the interior solution. On the other hand, the fixed solar wind values were used at these boundaries for the simulations done in the corotating frame to better enforce the solid rotation of the solar wind relative to the computational box.

[21] The plasma conditions near Uranus are not well known, making the setting of inner boundary conditions 
Table 1. Data Used for Modeling

\begin{tabular}{|c|c|}
\hline Parameter & Value \\
\hline \multicolumn{2}{|c|}{ Uranus Data } \\
\hline Mass & $8.6832 \times 10^{25} \mathrm{~kg}$ \\
\hline Radius $\left(\mathrm{R}_{\mathrm{U}}\right)$ & $25,559 \mathrm{~km}$ \\
\hline Rotation period & $17.24 \mathrm{~h}$ \\
\hline Obliquity & $97.9^{\circ}$ \\
\hline Magnetic dipole moment & $22,836 \mathrm{nT} \mathrm{R}_{\mathrm{U}}^{3}$ \\
\hline $\begin{array}{l}\text { Angle between the magnetic } \\
\text { and rotation axes }\end{array}$ & $58.61^{\circ}$ \\
\hline Dipole shift along the rotation axis & $0.310 \mathrm{R}_{\mathrm{U}}$ \\
\hline \multicolumn{2}{|c|}{ Solar Wind Data } \\
\hline Solar wind velocity & $450 \mathrm{kms}^{-1}$ \\
\hline Plasma density & 0.05 and $0.1 \mathrm{amu} / \mathrm{cm}^{3}$ \\
\hline Plasma temperature & $54,541 \mathrm{~K}(4.7 \mathrm{eV})$ \\
\hline \multicolumn{2}{|c|}{ Inner Boundary Conditions at $r=3 R_{U}$} \\
\hline Plasma density & $0.4 \mathrm{amu} / \mathrm{cm}^{3}$ \\
\hline Plasma temperature & $50,000 \mathrm{~K}$ \\
\hline
\end{tabular}

difficult. For the models presented here, we assume that the plasma is rigidly corotating with the planet to at least to a few planetary radii. We apply rigid corotation at our inner boundary at $3 \mathrm{R}_{\mathrm{U}}$. Taking the inner boundary at the surface of the planet would require finer and more grid cells, which would slow down the computation. On the basis of our experience with modeling the terrestrial magnetosphere we feel that placing the inner boundary at $3 \mathrm{R}_{\mathrm{U}}$ is an acceptable approximation. We take the temperature and the density at the inner boundary to be $50,000 \mathrm{~K}$ (similar to the solar wind value) and $0.4 \mathrm{amu} / \mathrm{cm}^{3}$ (which corresponds to a 4 times compression of the $0.1 \mathrm{amu} / \mathrm{cm}^{3}$ solar wind density due to a strong shock), respectively. In our exploratory simulations various densities were prescribed at the inner boundary, but they did not seem to have a significant effect on the structure of the magnetosphere.

[22] The magnetic field $\mathbf{B}$ is split into a dipole component $\mathbf{B}_{0}$ and the deviation field $\mathbf{B}_{1}=\mathbf{B}-\mathbf{B}_{0}$ [see, e.g., Ogino and Walker, 1984; Tanaka, 1994]. This splitting does not assume that $\mathbf{B}_{1}$ is small, but the discretized form of the split MHD equations allows more accurate numerical representation of the dipole field near the planet. In particular the splitting allows us to impose very simple boundary conditions at the inner boundary. We allow $\mathbf{B}_{1}$ to float, but it remains negligible relative to $\mathbf{B}_{0}$. In case of the time accurate simulation $\mathbf{B}_{0}$ depends on time due to the rotation of Uranus. The time derivative of $\mathbf{B}_{0}$ is disretized as a source
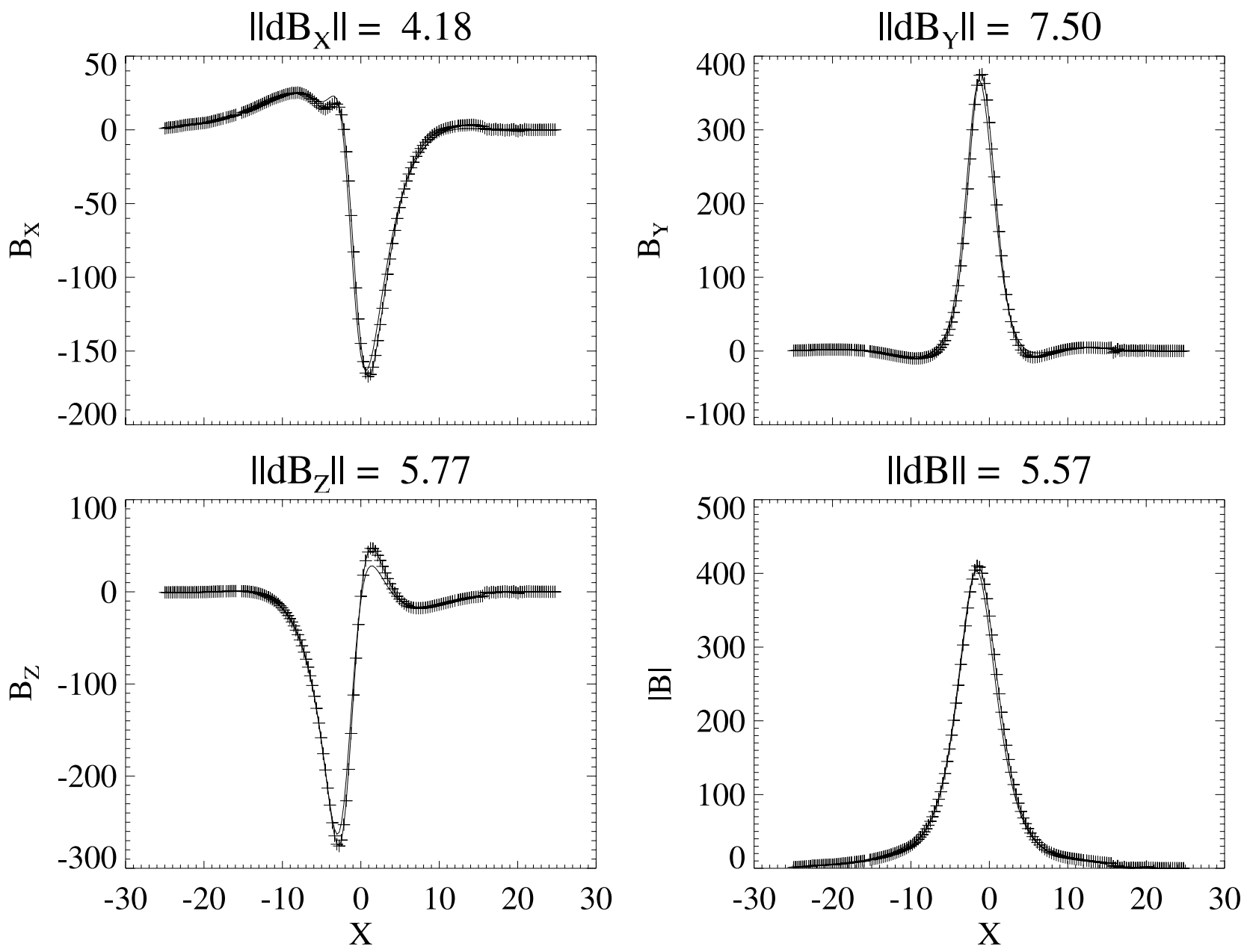

Figure 3. Comparison of the measured (crosses) and the fitted offset dipole (continuous line) magnetic fields in the UGR frame near Uranus. The small jump at $x \approx 15 \mathrm{R}_{\mathrm{U}}$ marks the crossing of the magnetopause. 

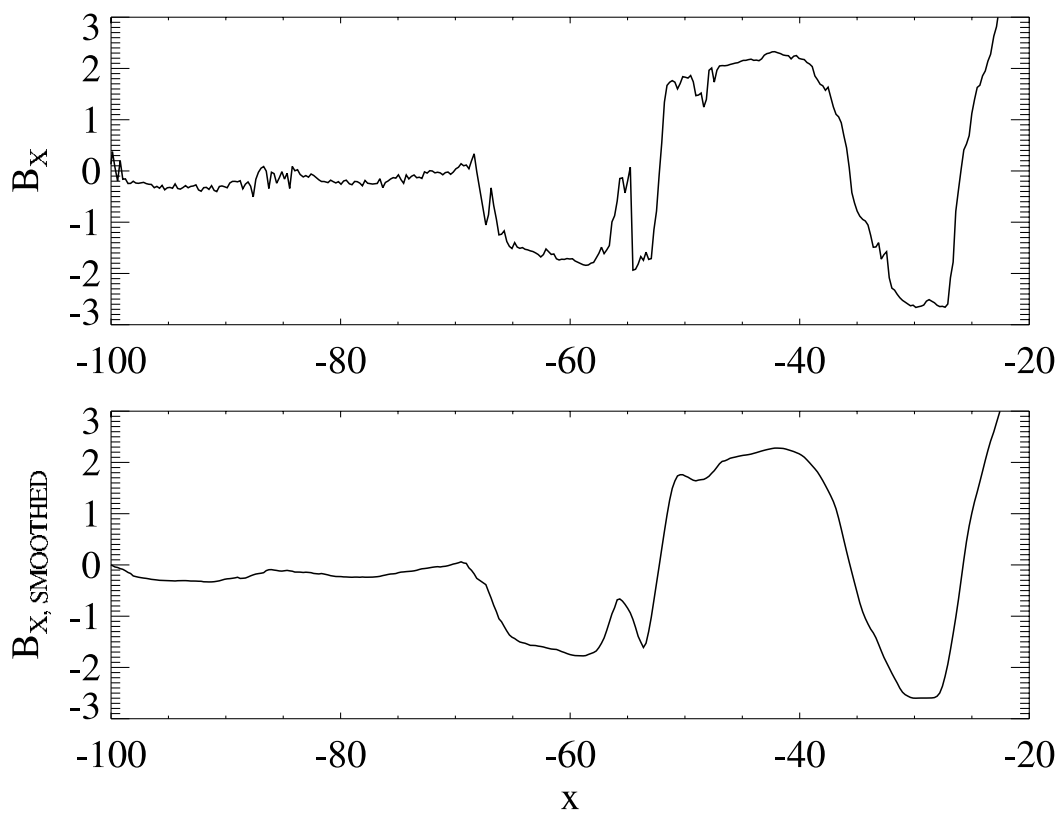

Figure 4. Variation of $\mathbf{b}_{x}$ in the 48-s averaged Voyager data set (top) and in the 80-min smoothed data used for comparisons with the simulations in the magnetotail.

term in the induction equation, and it is integrated with the implicit scheme.

\section{Voyager Data}

[23] Voyager 2 data were used to set the upstream solar wind conditions for the simulations. In this data set the magnitude of the magnetic field outside the bow shock was below $1 \mathrm{nT}$ on both the inbound and the outbound trajectories [Ness et al., 1986]. The individual components of the magnetic field show oscillations around zero with small amplitudes without any obvious average direction. Since the solar wind field is not known while Voyager was inside the magnetosphere of Uranus, given the available observations it seems reasonable to take the solar wind magnetic field to be negligible during the flyby. This is rather fortunate

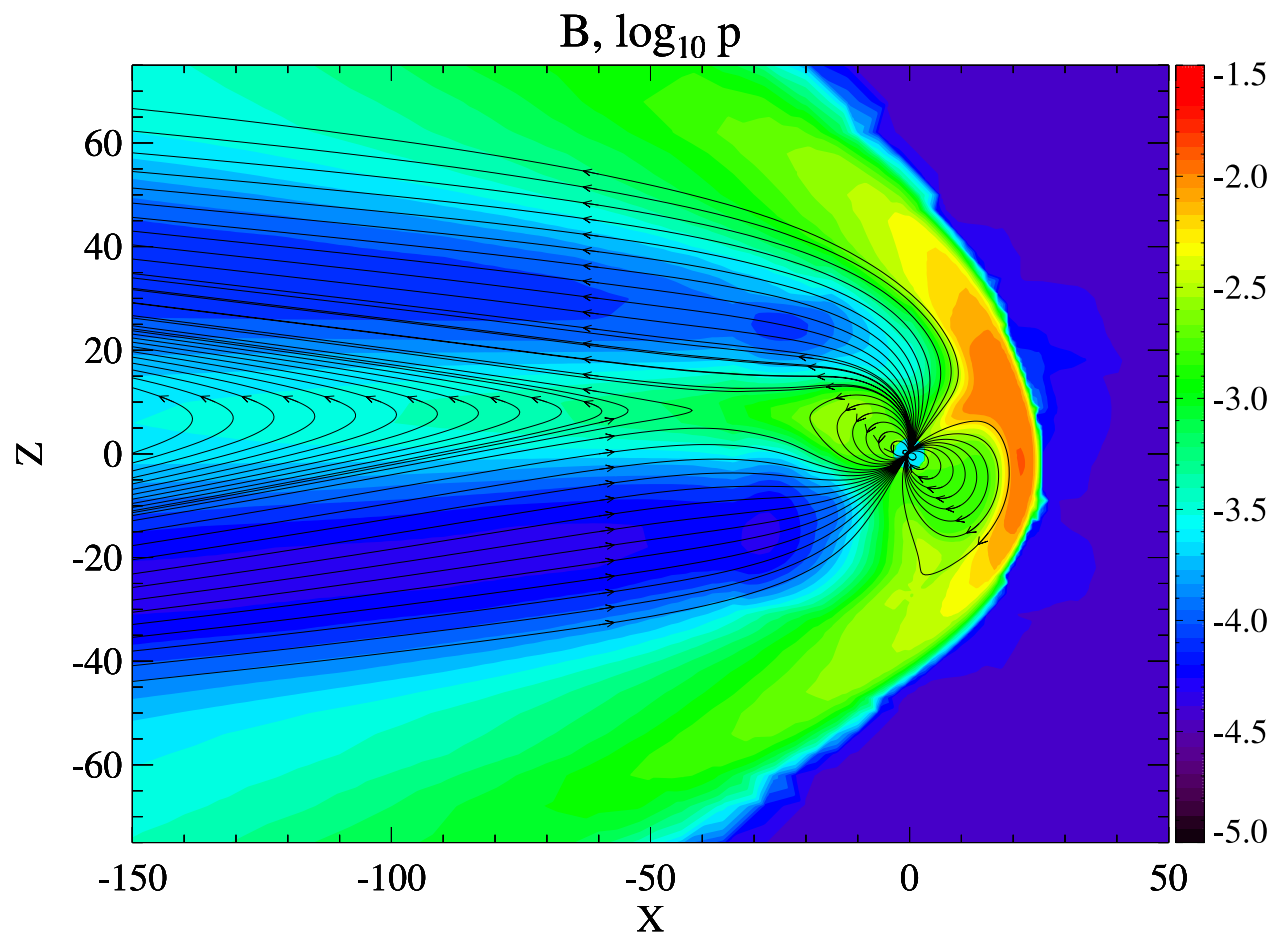

Figure 5. The magnetic field lines and the logarithm of pressure (in $\mathrm{nPa}$ ) in the inertial stationary approximation, in the $X-Z$ plane. Note the asymmetric shape of the bow shock. 

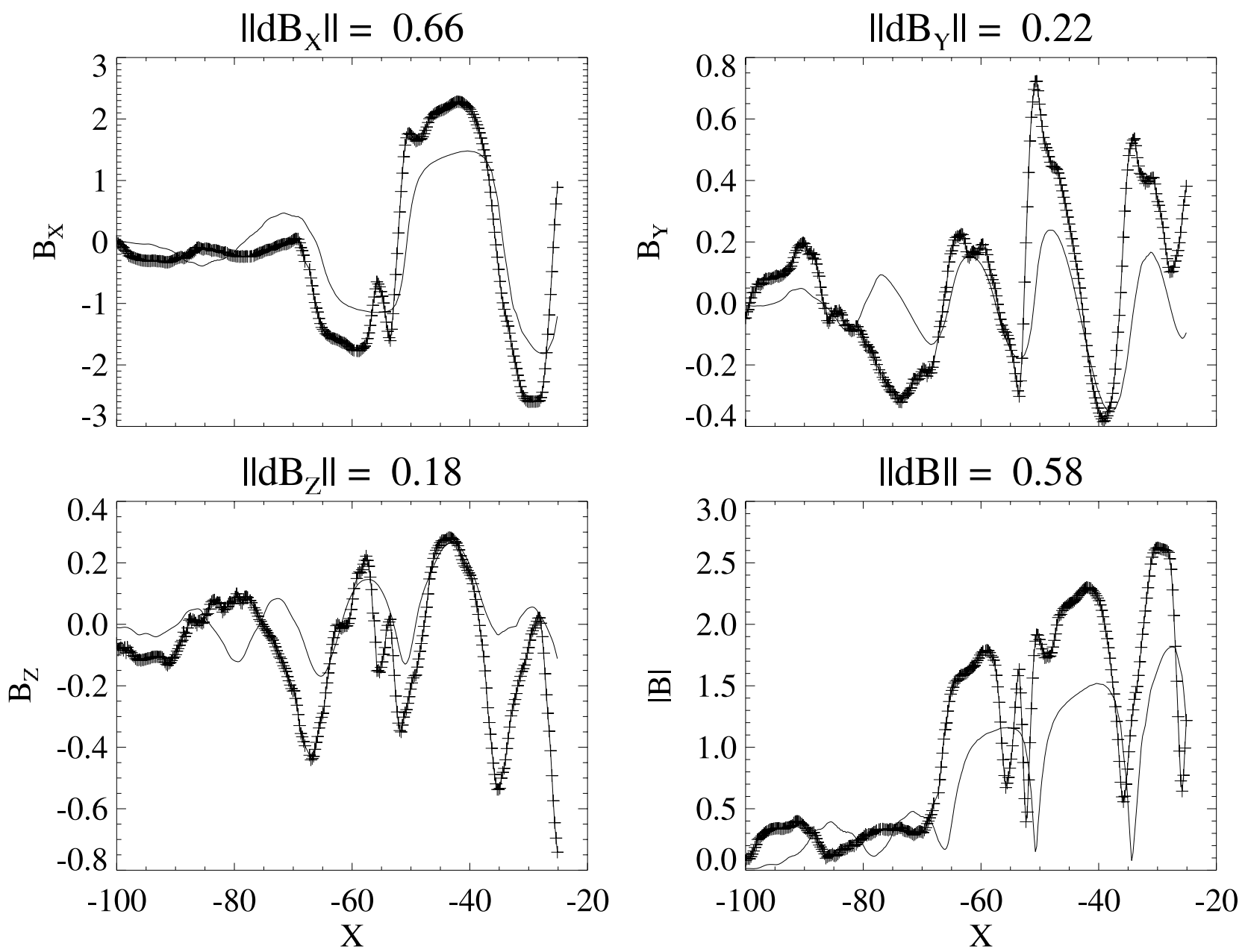

Figure 6. Comparison of the measured (crosses) and simulated (continuous line) data series in the magnetotail for the inertial stationary simulation. Above the figures are the RMS differences between the two data sets in nT. Note the phase shift between the curves due to approximations made in this model.

because it allows us to obtain a steady state solution in the corotating frame. If the interplanetary magnetic field was not negligible, it would break the cylindrical symmetry of the solar wind. Although in the time-accurate simulations we do not need the symmetry of zero magnetic field, we choose to retain the zero value used in the corotating simulations.

[24] Voyager plasma data were used to set the velocity, density, and temperature of the solar wind. In each of our simulations the solar wind flowed exactly from the positive direction of the $X_{U S O}$ axis, with the velocity and temperature taken from Bridge et al. [1986]. The values used are listed in Table 1. Our initial simulations used the nominal Voyager density of $0.05 \mathrm{amu} / \mathrm{cm}^{3}$ for the plasma density, but later we experimented with $\rho_{S W}=0.1 \mathrm{amu} / \mathrm{cm}^{3}$ to obtain a better agreement with data in the magnetotail region. This increase of density is somewhat justified by examining the plasma data that shows approximately this density at the outbound bow shock crossing time when Voyager left the magnetosphere. Of course, we do not have direct knowledge of the solar wind parameters while Voyager was inside the magnetosphere.

[25] Values for the magnetic dipole moment and dipole tilt of Uranus can be found in the work of Ness et al. [1986] and Connerney et al. [1987]. Using these values as a starting point, we have fit the Voyager data in USO coordinates to the field due to an offset, tilted dipole. We retain the dipole magnitude and tilt from the literature and vary the offset and orientation to obtain the best fit, shown in Figure 3. The dipole parameters used in the simulations are given in Table 1 .

[26] In order to compare the Voyager data with the simulations, we need to transform it from its native coordinate system (USO) to the USM coordinate system. This step requires us to know a certain instant of time when the two frames coincided. The process of fitting the dipole orientation and offset to the Voyager data also allows the determination of the coincidence time. We found that one of these coincidences must have occurred at 0 hours $27 \mathrm{~min} 22 \mathrm{~s}$ on 23 January 1986.

[27] Our comparison with the Voyager data is carried out using different methods for each of two distinct regions. In the vicinity of the planet, where the magnetic field is strong, we sample the simulation every $8 \mathrm{~min}$ (480 s) along the Voyager trajectory. This spacing is the most reasonable given the model resolution. The Voyager magnetic field data contains 48-s averages of the measured quantities, so in this region we compare our 

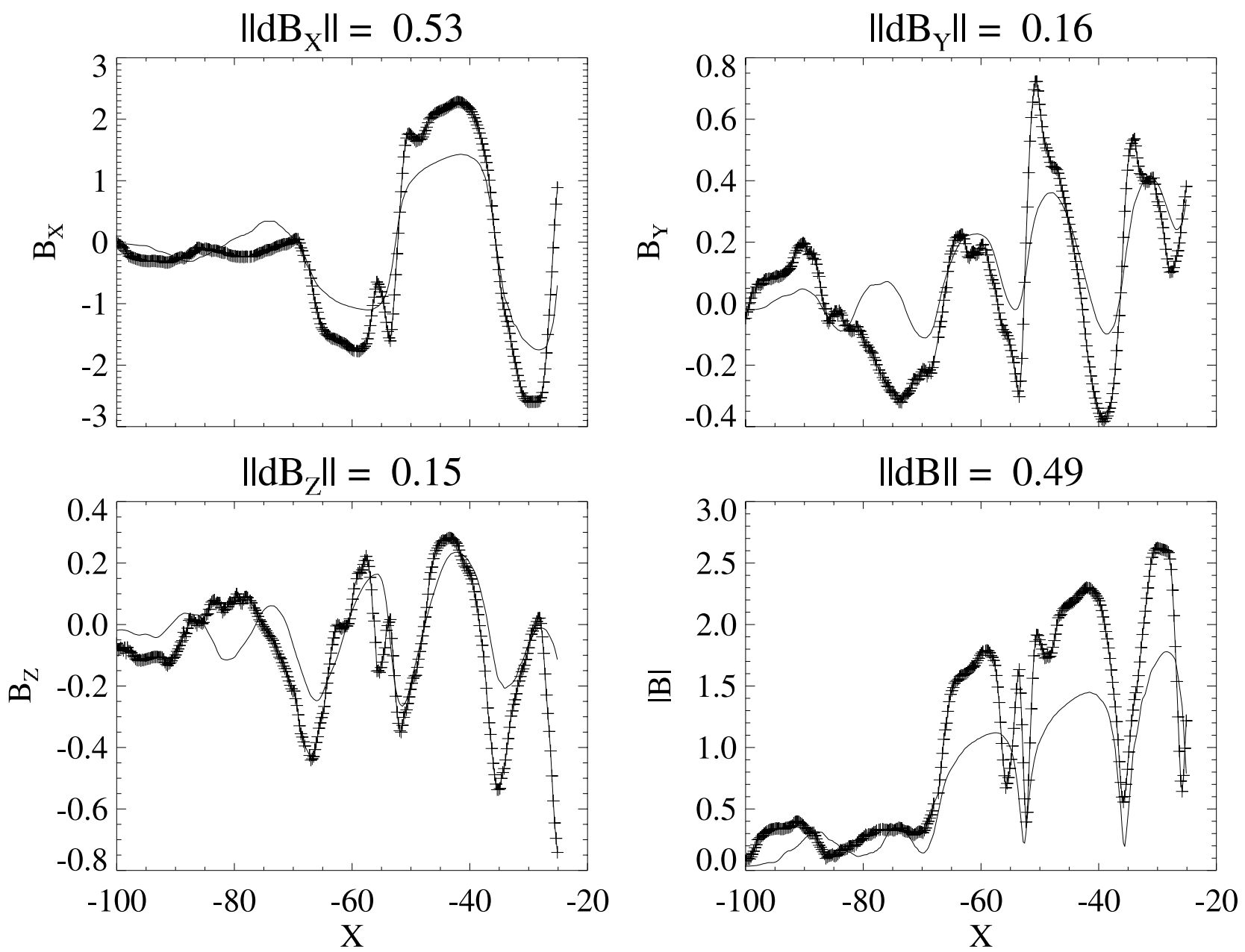

Figure 7. Comparison of the measured (crosses) and simulated (continuous line) data series in the tail region for the first corotating simulation with $0.05 \mathrm{amu} / \mathrm{cm}^{3}$ for solar wind plasma density. The phase shift seen in the inertial steady state simulation (Figure 6) has disappeared.

simulation with every 10th Voyager magnetic field measurement. When Voyager is in the magnetotail, the small magnitude of the magnetic field, the observed noise, and the large scale lengths suggest that smoothing the original data would be beneficial. We apply an 80-min sliding average to the original data; thus in the magnetotail every point extracted from the simulation is compared with the average of the data in an $80 \mathrm{~min}$ interval. Figure 4 shows the original and smoothed observational data for the $\mathbf{B}_{x}$ component, as an example.

\section{Simulation Results}

\subsection{Inertial Stationary Approximation}

[28] As a zeroth order approximation, we model Uranus as a nonrotating sphere with a fixed orientation of its magnetic dipole. Although this arrangement is highly simplified, the steady state solution obtained in this nonrotating system can be rotated around the $X_{U S O}$ axis, thus making comparison with the Voyager data possible. This procedure, however, is only a crude approximation, since the inertial forces and the relative rotation of the solar wind were neglected during the simulation. The inertial stationary solutions are useful to explore the basic size and shape of the magnetosphere of Uranus and also serve as a baseline for the more complex corotating simulations.

[29] In this simulation the magnetic dipole axis lies in the $X-Z$ plane tilted by $31.39^{\circ}$ toward the Sun relative to the $Z$ axis and it is offset by $-0.31 \mathrm{R}_{\mathrm{U}}$ along the $X$ axis. The solar wind density is taken to be the nominal $0.5 \mathrm{amu} / \mathrm{cm}^{3}$. The smallest cells are $0.125 \mathrm{R}_{\mathrm{U}}$ at the maximum resolution resulting in around 8300 blocks or half million cells.

[30] We use the local time stepping with explicit time discretization to obtain a steady state solution after 7000 iterations. The solution is shown in Figure 5. It is interesting how the strong tilt of the dipole makes the bow shock asymmetric. The magnetic field lines are reconnecting at around $-30 \mathrm{R}_{\mathrm{U}}$. As in any numerical MHD model, the location of the $\mathrm{X}$ point depends on the numerical resistivity and it may differ significantly from the true location, however this has relatively small effect on the magnetic field away from the reconnection site. The measured and simulated data series in the magnetotail are compared in Figure 6. The plasma sheet crossings are marked by the jumps in the magnetic field components. Although the rotation of the dipole was neglected in this simulation, the agreement of the numerical solution and the observa- 

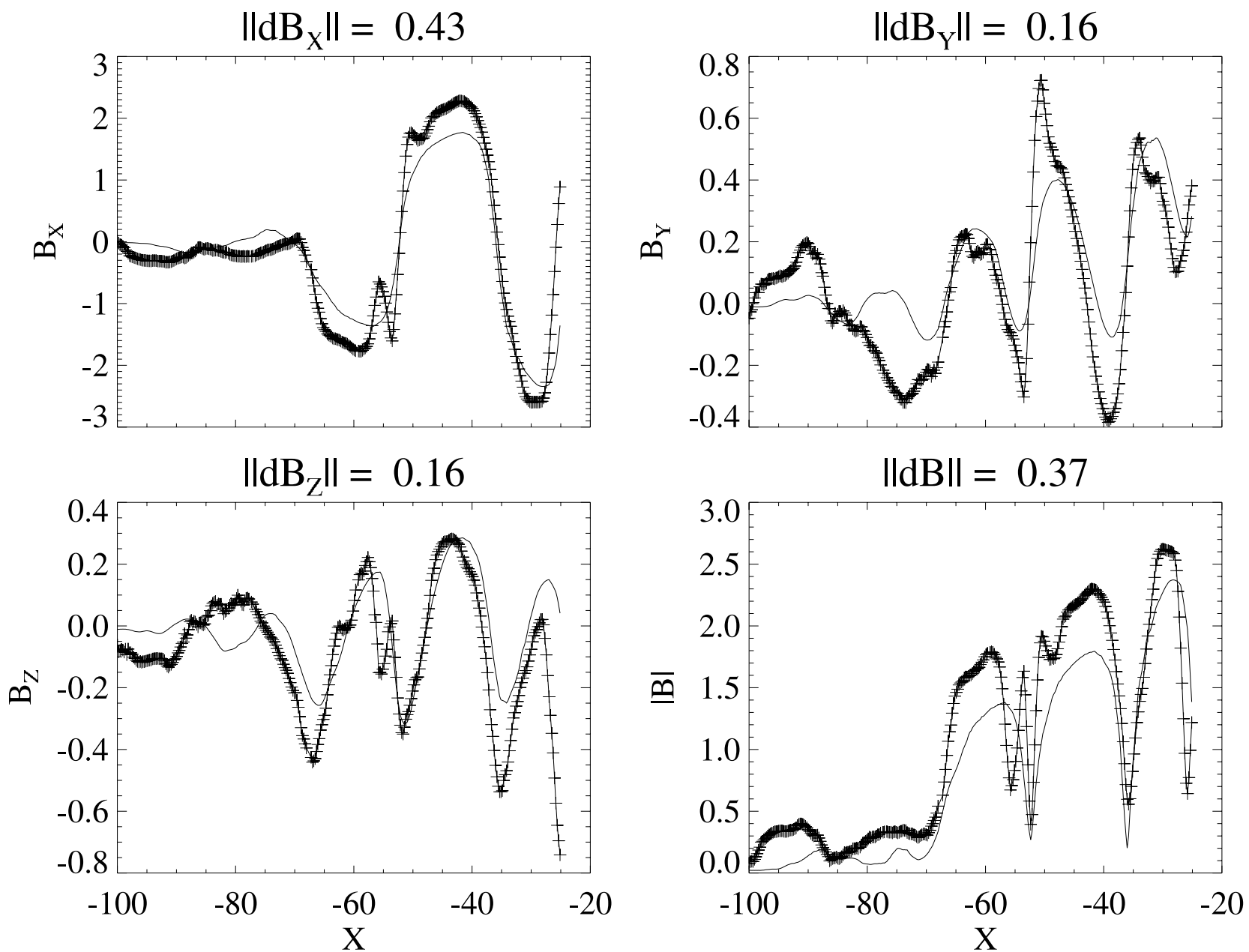

Figure 8. Comparison of the measured (crosses) and simulated (continuous line) data series in the tail region for the second corotating simulation with $0.1 \mathrm{amu} / \mathrm{cm}^{3}$ solar wind plasma density. Note the improved agreement of magnitudes relative to Figure 7.

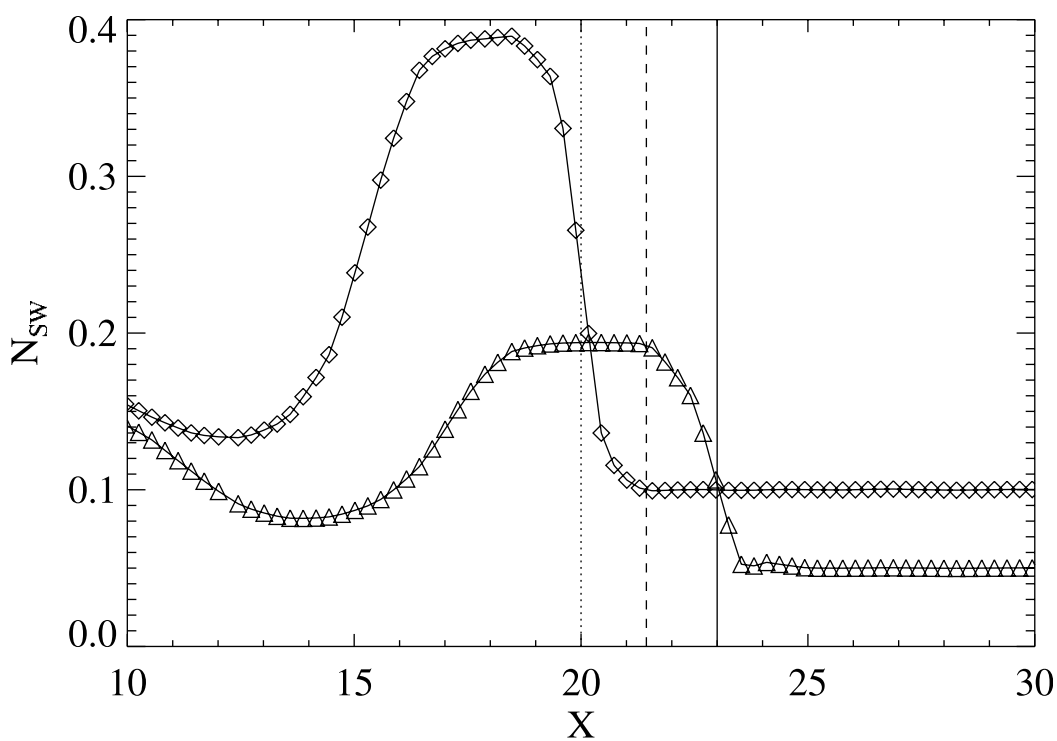

Figure 9. Density variation along the Voyager trajectory in two corotating simulations with $0.05 \mathrm{amu} / \mathrm{cm}^{3}$ (triangles) and $0.1 \mathrm{amu} / \mathrm{cm}^{3}$ (diamonds) solar wind density, respectively. The position of the simulated bow shocks are marked by the continuous and dotted vertical lines, while the dashed line shows the position of the bow shock as measured by Voyager at around $21.4 \mathrm{R}_{\mathrm{U}}$ in the $X_{U S M}$ coordinate. 


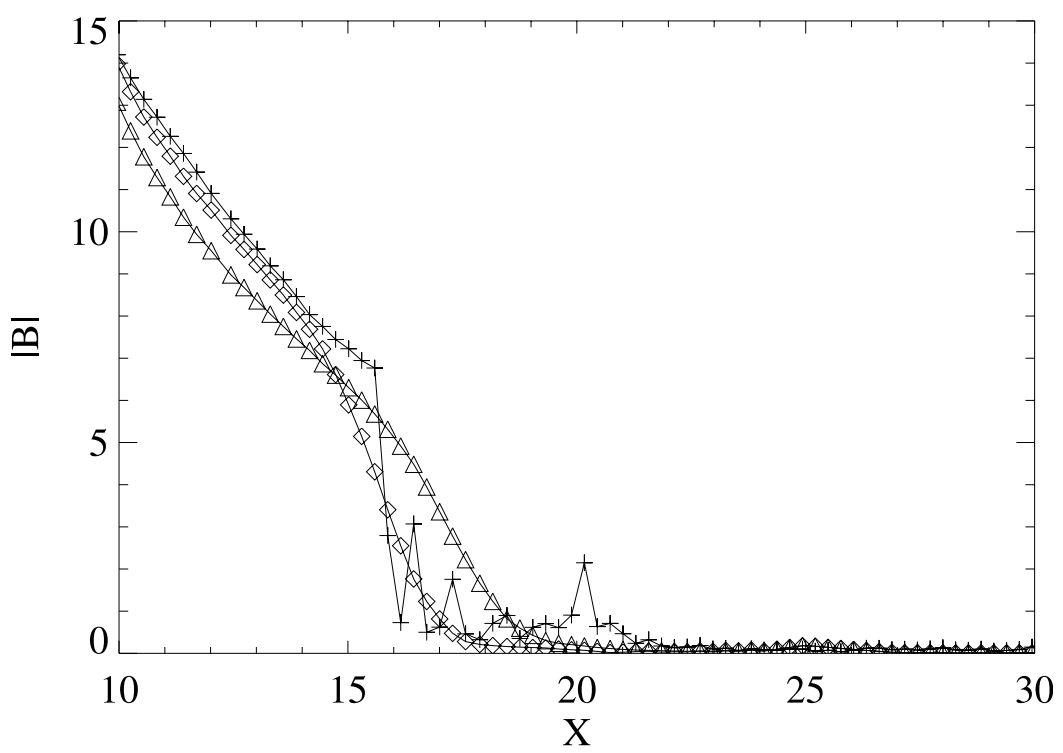

Figure 10. The magnitude of the magnetic field as Voyager crosses the magnetopause inbound. The results from the first (triangles) and second (diamonds) corotating simulations are compared with the observed values (crosses). Note the good agreement between the second simulation and the measured data.

tions is surprisingly good. The discrepancy in magnitudes is significant, but the most interesting feature is that the two data series are shifted relative to each other. We interpret this shift as the lack of twisting of the magnetotail in the numerical solution. As the following models show, performing the simulation in a corotating frame which correctly includes the relative rotation of the solar wind and the inertial forces will improve the agreement of numerical and observational results. The worst agreement is in the $\mathrm{X}$ component of the magnetic field, which leads to an underestimate of the magnetic field magnitude. To quantify the difference between the simulations and the observations, we have calculated the root-mean-square (RMS) of the difference for all the components and also for the magnitude of the magnetic field. The RMS errors are given in units of $\mathrm{nT}$ above the plots.

\subsection{Corotating Frame}

[31] The corotating simulations are carried out in the USM coordinate system. The tilt of the rotation axis relative

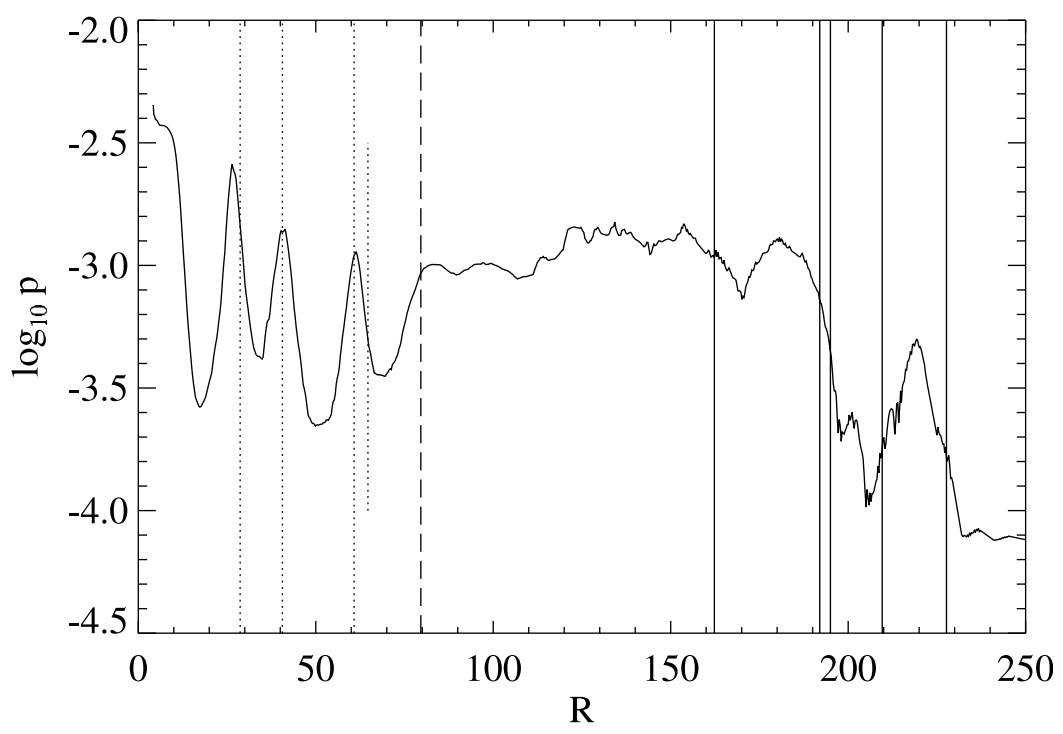

Figure 11. The logarithm of plasma pressure (in $\mathrm{nPa}$ ) in the magnetotail for the second corotating simulation as a function of the planetocentric distance. The vertical lines mark the observed [Ness et al., 1986, Table 1] current sheet crossings (dotted), magnetopause crossing (dashed), and bow shock crossings (continuous). The partial current sheet crossing at $R=64.7$ is shown as a shorter dotted line. See the discussion in the text. 

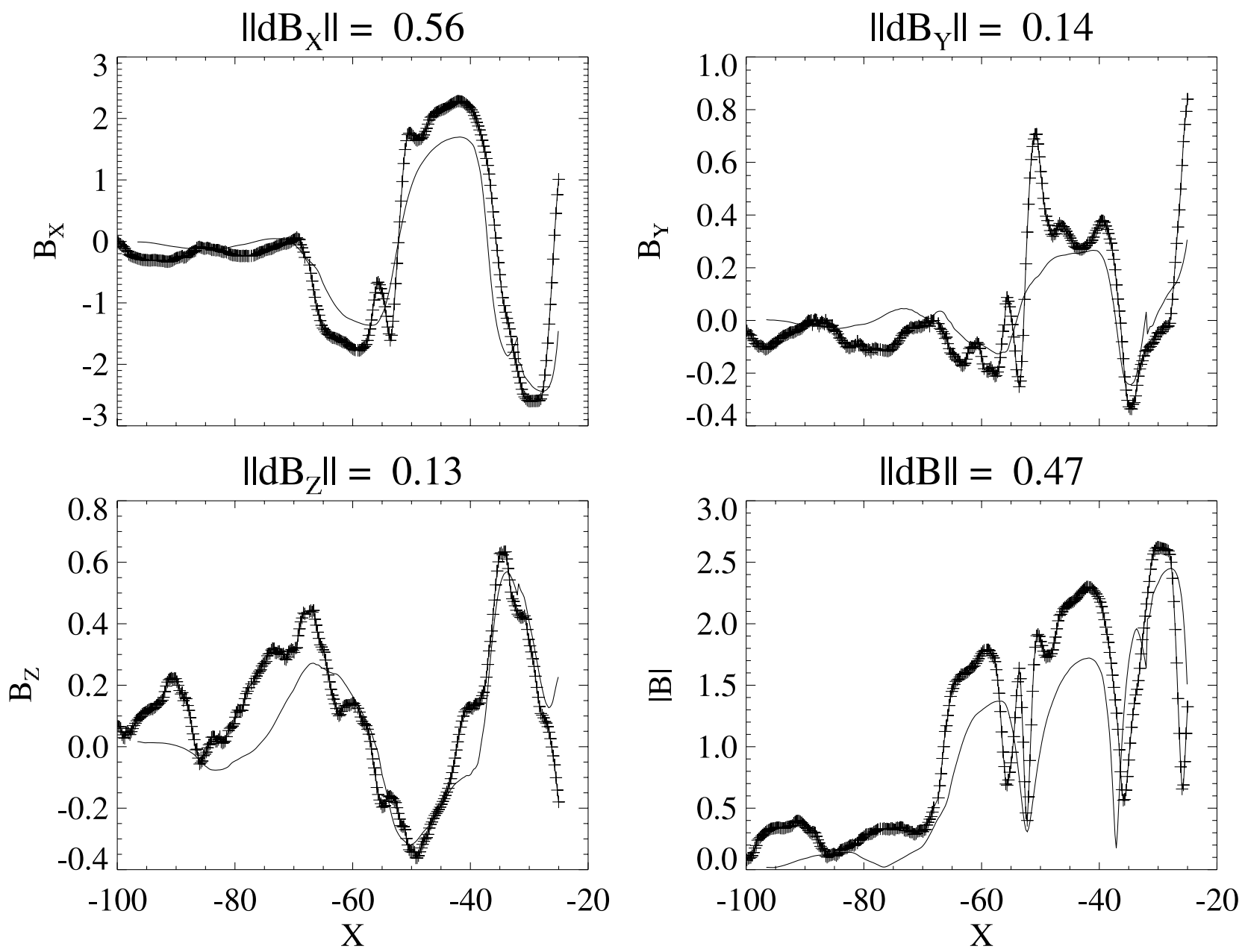

Figure 12. Comparison of the measured and simulated data series in the tail region for the time-accurate simulation in the USO frame. Note that the $B_{y}$ and $B_{z}$ components are rotated relative to the $B_{y}$ and $B_{z}$ components obtained in the corotating simulations in the USM frame.

to $X_{U S M}$ is neglected; thus the angular velocity $\Omega=(\Omega, 0,0)$ is taken to be parallel with the $X_{U S M}$ axis and the radial position vector $\mathbf{d}=(0, y, z)$. In the corotating simulations the Coriolis and centrifugal forces are properly taken into account in equations (2) and (4). Because we are in the rotating frame, special care must be taken when applying boundary conditions on the walls of the simulation domain. In the corotating frame the solar wind appears to rotate with $-\Omega$; thus at the boundaries (except for the outflow boundary) we prescribe the velocity as

$$
\mathbf{u}=-\boldsymbol{\Omega} \times \mathbf{d}+\mathbf{u}_{S W},
$$

where $\mathbf{u}_{S W}$ is the solar wind speed, which is parallel with the $X$ axis.

[32] The magnetic dipole is tilted by $31.39^{\circ}$ in the $X-Z$ plane and it is offset by $-0.31 \mathrm{R}_{\mathrm{U}}$ along the $X$ axis. The smallest cells are $1 / 8 \mathrm{R}_{\mathrm{U}}$, as in the inertial simulation. The steady state solution is obtained with local time stepping in 7000 iterations.

[33] Here we report the results from two simulations. In the first simulation the solar wind density is taken to be $0.05 \mathrm{amu} / \mathrm{cm}^{3}$, while in the second simulation $0.1 \mathrm{amu} / \mathrm{cm}^{3}$ is used. First we compare the simulated and observed magnetic field data in the tail region. Figure 7 shows the results for the first corotating simulation with $\rho_{S W}=$ $0.05 \mathrm{amu} / \mathrm{cm}^{3}$. There is a marked improvement relative to the inertial steady state results in Figure 6. The phase differences disappeared, and the root mean square deviations also decreased in all three components. We attribute the improvement to the proper inclusion of the relatively rotating solar wind and the inertial forces, which result in a twisted magnetotail.

[34] The result obtained with the second corotating simulation with $\rho_{S W}=0.1 \mathrm{amu} / \mathrm{cm}^{3}$ is shown in Figure 8. The difference between data and simulation is further reduced. The most significant change is in the amplitude of the $B_{x}$ component. The increased solar wind density compresses the magnetosphere more than in the previous case and increases $B_{x}$. This results in much better agreement with the data: the RMS error of $B_{x}$ decreased from $0.53 \mathrm{nT}$ to $0.43 \mathrm{nT}$.

[35] The increased solar wind density and the resulting compression of the magnetosphere can be clearly seen in the change of position of the bow shock and the magnetopause. In Figures 9-11 we compare the positions of the bow shock and the magnetopause in the two simulations with the observations [Ness et al., 1986]. The positions of the 


\section{time $=3.00$ Uday}

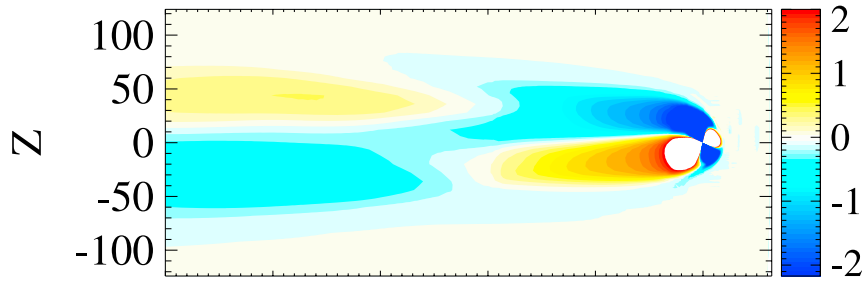

time $=3.25$ Uday

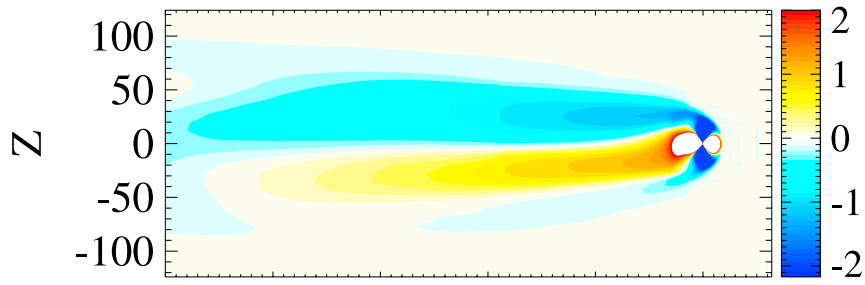

time $=3.50$ Uday

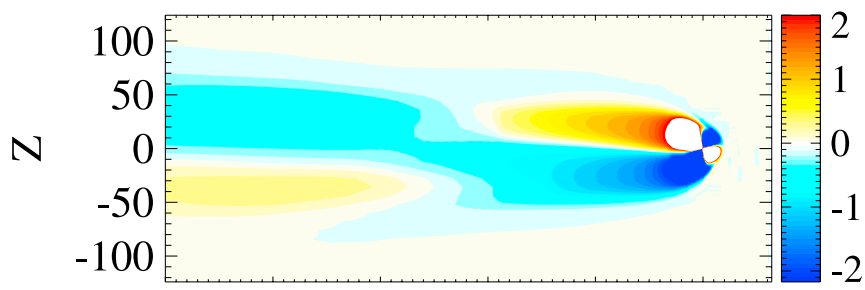

time $=3.75$ Uday

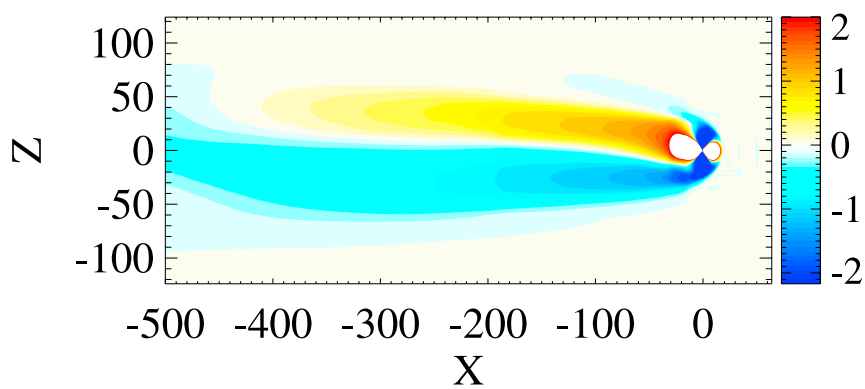

\section{time $=4.00$ Uday}

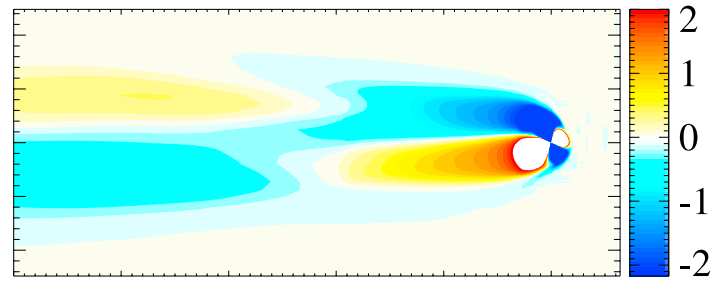

time $=4.25$ Uday

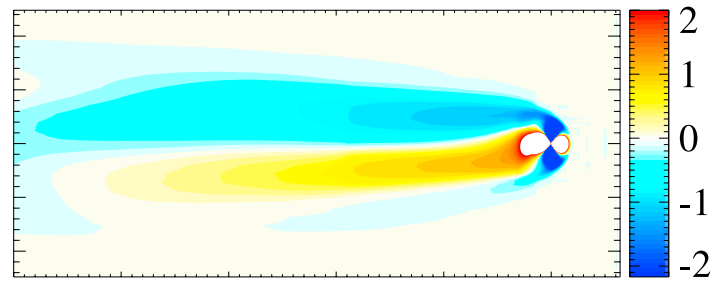

time $=4.50$ Uday

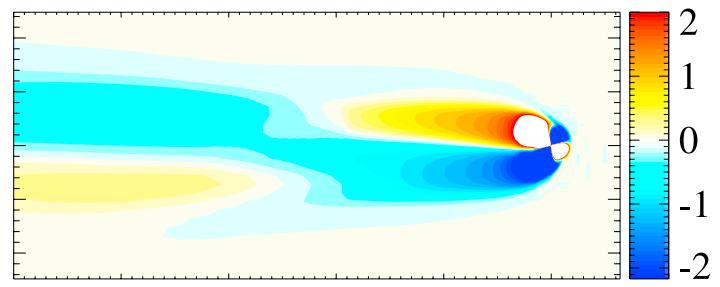

time $=4.75$ Uday

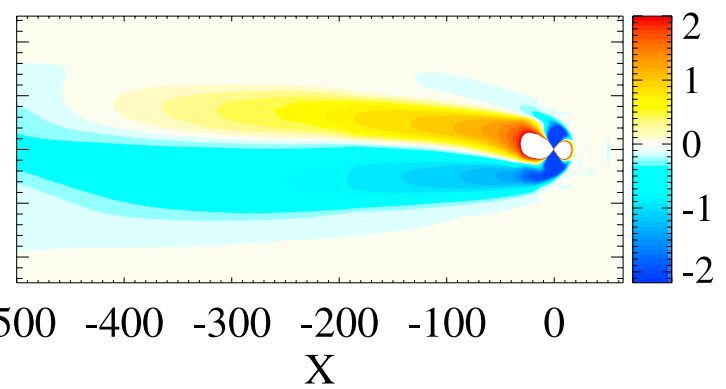

Figure 13. The $\mathrm{x}$ component of the magnetic field in the $Y_{U S O}=0$ plane. The color range is saturated (white) near the planet. The panels are separated by a quarter rotation periods on days 3 and 4 during the time-dependent simulation. This time series displays the periodicity of rotation.

inbound bow shock crossing are shown in Figure 9. The width of the shocks in the numerical results is due to the finite grid resolution, which is $1 \mathrm{R}_{\mathrm{U}}$ in the vicinity of the inbound bow shock crossing. The bow shock is captured with three grid cells, which is typical of high-resolution shock-capturing schemes. The extracted trajectory contains about six to eight points across the shock, which results from the selected 8 min time intervals between the extracted data points and the roughly $30 \mathrm{~km} / \mathrm{s}$ speed of the space craft which corresponds to about $0.5 \mathrm{R}_{\mathrm{U}}$ distance between the data points. The trajectory is not perpendicular to the bow shock which makes the apparent width larger.

[36] We take the position of the shock to be in the middle of the steep slopes, at around $x=23 \mathrm{R}_{\mathrm{U}}$ and $x=20 \mathrm{R}_{\mathrm{U}}$ for the two simulations, respectively. The observations showed that the first crossing occurred at $21.4 \mathrm{R}_{\mathrm{U}}$, which is in between the two simulation results.

[37] The positions of the inbound magnetopause crossing can be read from the magnitude of the magnetic field shown in Figure 10. The observed data has a sharp jump at $x \approx$ $16 \mathrm{R}_{\mathrm{U}}$ which corresponds to the magnetopause. In the simulation results the magnetopause is somewhat smeared out (which is difficult to avoid for a noncompressive discontinuity). If we take the middle of the sharp slopes, the magnetopause is at around $x=17 \mathrm{R}_{\mathrm{U}}$ and $x=16 \mathrm{R}_{\mathrm{U}}$ in the two simulations, respectively. The agreement of the observed and simulated positions is somewhat better for the second simulation with $\rho_{S W}=0.1 \mathrm{amu} / \mathrm{cm}^{3}$. 

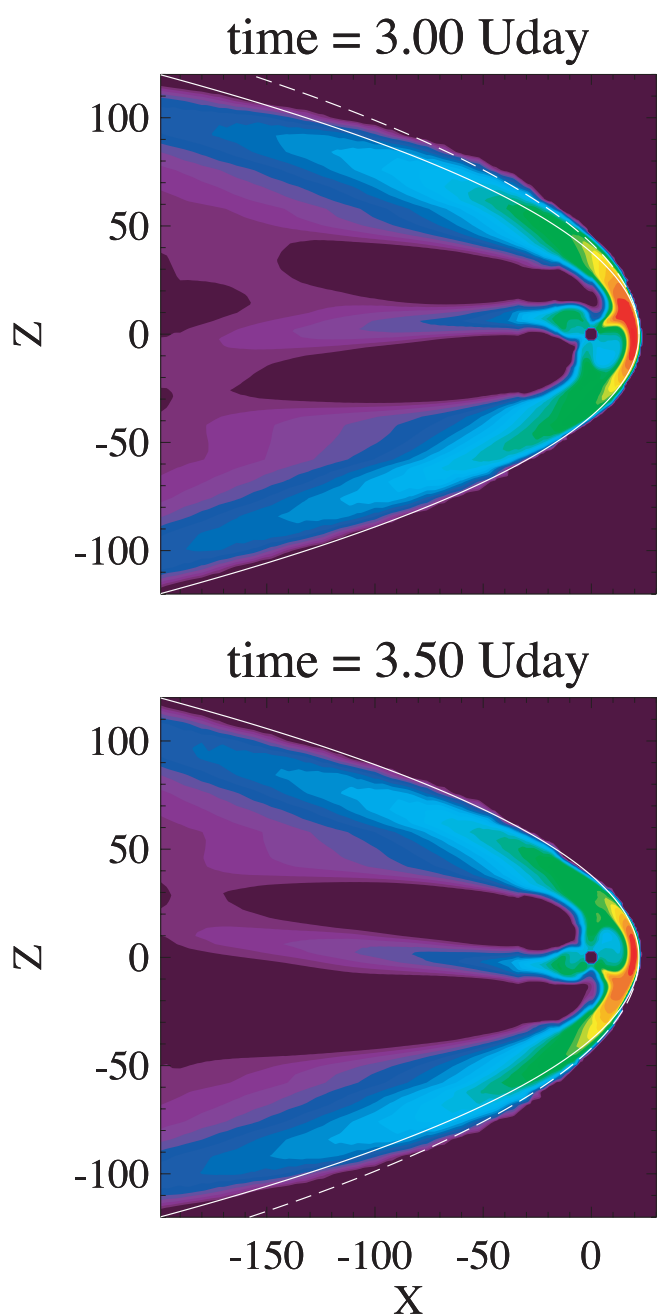

$$
\text { time }=3.25 \text { Uday }
$$
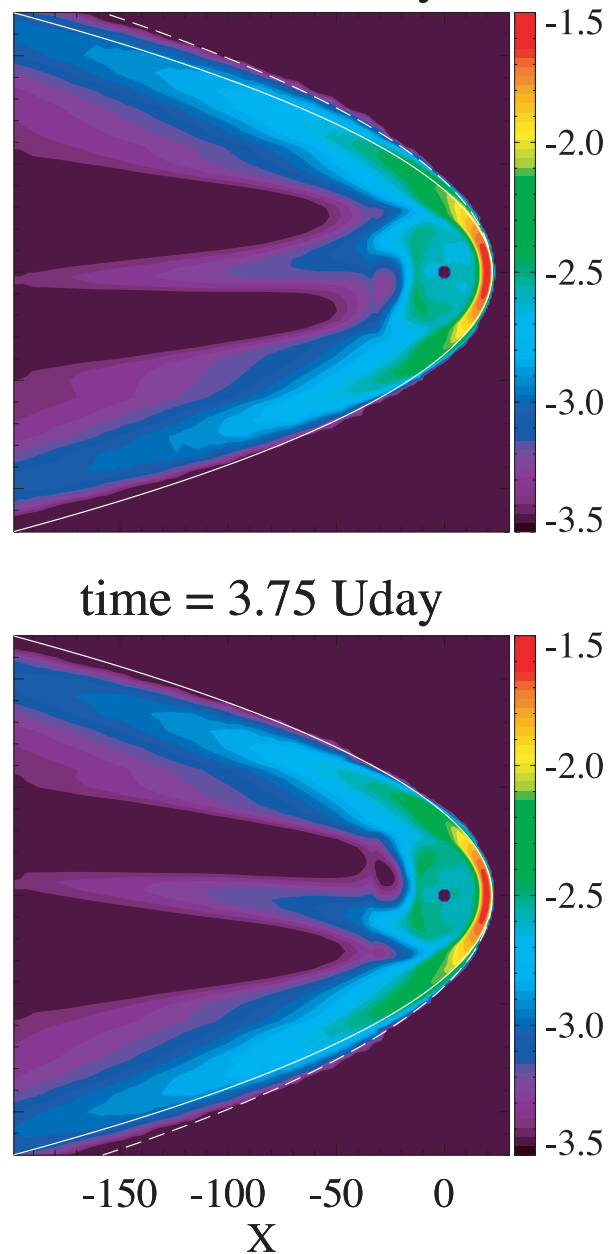

Figure 14. The logarithm of pressure is shown in the $10^{-3.5} \mathrm{nPa}$ to $10^{-1.5} \mathrm{nPa}$ range for four different times in the $X-Z$ plane of the USO frame. The full parabola fitted to the whole bow shock obeys $x=22-$ $z^{2} / 65$ (continuous line), while the half parabola fitted to the bulge obeys $x=22-z^{2} / 80$ (dashed line).

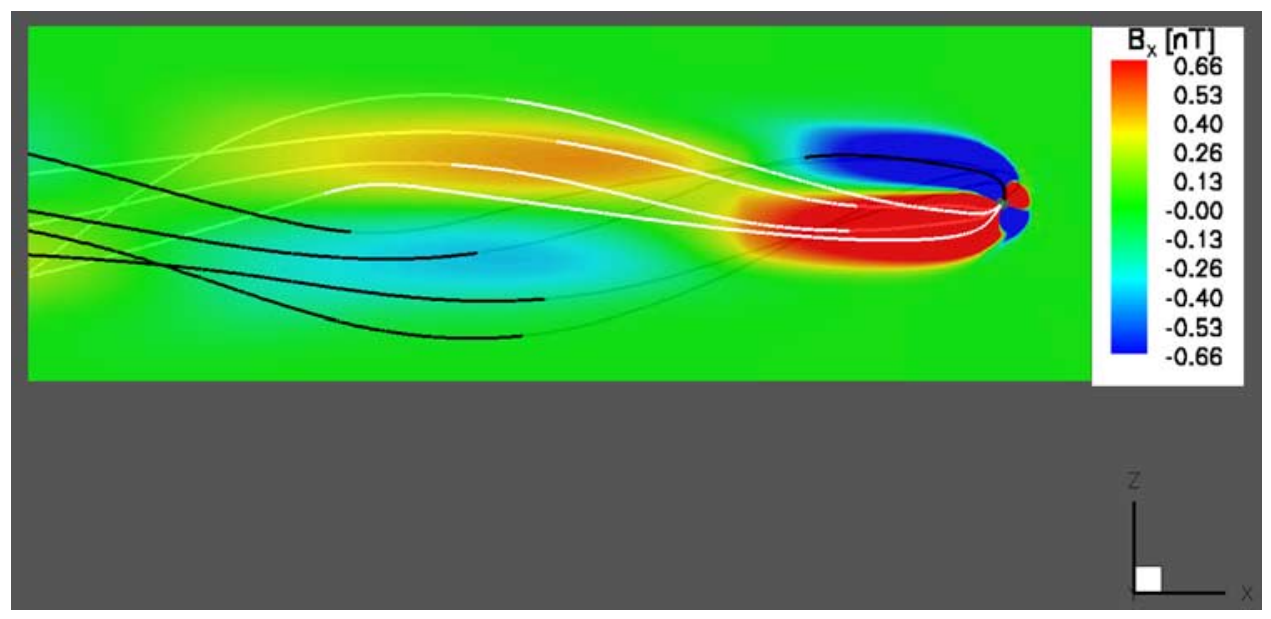

Figure 15. Magnetic field lines connected to the northern (black lines) and southern (white lines) poles. The $B_{x}$ component (colors) shows the topology of the magnetic field in the $X-Z$ plane. Note that the color scale is saturated near the planet. 


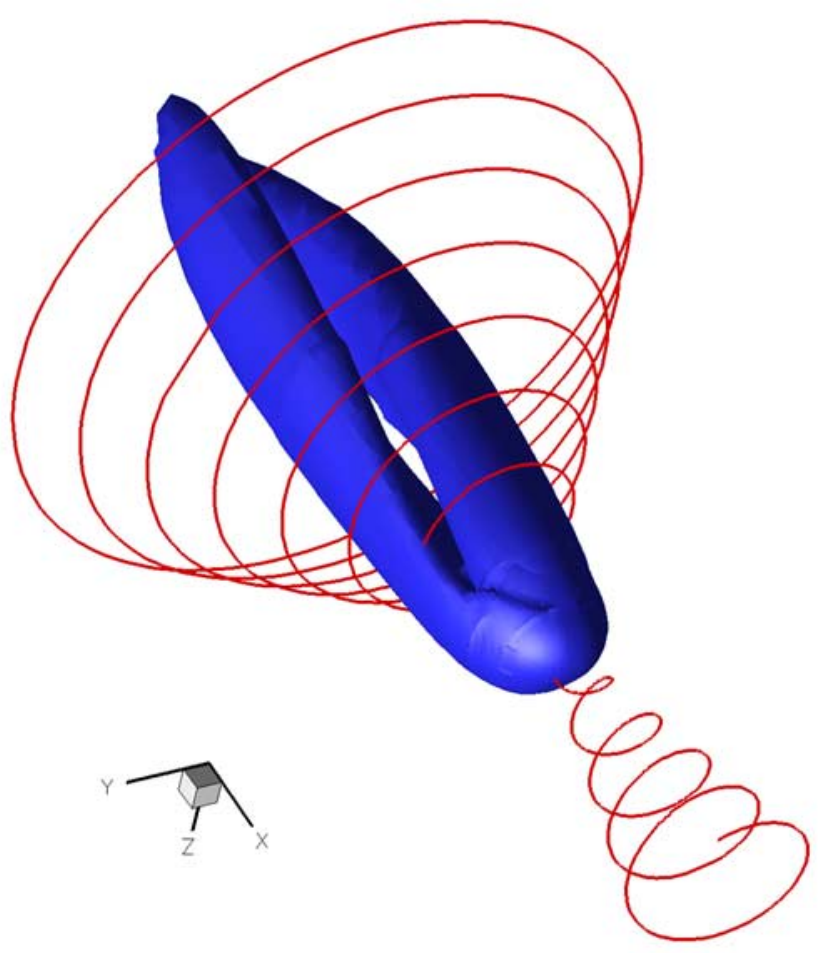

Figure 16. Three-dimensional visualization of the magnetopause as a blue isosurface at $|B|=0.5 \mathrm{nT}$. The Voyager trajectory outside the magnetopause is shown as a red line in the USM frame. Both the inbound and outbound magnetopause crossings are visible. Note the twist of the lobes.

[38] The outbound current sheet, magnetopause, and bow shock crossings for the second corotating simulation are shown in Figure 11. This plot directly compares the observed crossings [Ness et al., 1986, Table 1] with the simulation results. The large oscillations in the pressure from $x=0 \mathrm{R}_{\mathrm{U}}$ to $x=-68 \mathrm{R}_{\mathrm{U}}$ result from the Voyager trajectory passing through the rotating magnetotail. The observed current sheet crossings coincide nicely with the pressure maxima up to the planetocentric distance $R=$ $70 \mathrm{R}_{\mathrm{U}}$. Only the partial current sheet crossing at $R=64.7$ has no corresponding pressure peak (see discussion below). The location of the magnetopause crossing can be identified as the point where these oscillations disappear at around $R=$ $80 \mathrm{R}_{\mathrm{U}}$. The observed outbound magnetopause crossing at $R=79.6 \mathrm{R}_{\mathrm{U}}$ almost perfectly coincides with that. Since the bow shock is somewhat smeared out in the numerical solution, the bow shock crossings appear as sharp gradients in the pressure. The observed bow shock crossings nicely coincide with the sudden gradients in the pressure, and the final bow shock crossing observed at $x=-187.6 \mathrm{R}_{\mathrm{U}}$ is in excellent agreement with the simulation.

[39] Regarding the partial crossing of the current sheet, Hammond et al. [1990] speculated that it may be due to the curvature of the current sheet which changes with the tilt of the magnetic dipole. The dipole tilt varies (approximately) between $22^{\circ}$ and $38^{\circ}$ relative to the $Z_{U S M}$ axis due to the $8^{\circ}$ tilt of the rotation axis relative to the $X$ axis. For sake of comparison, two nonrotating steady state simulations were carried out with $22^{\circ}$ and $38^{\circ}$ dipole tilts, respectively. We find that although the trend in the bending of the current sheet is similar to that shown in Figure 4 in the work of Hammond et al. [1990], the effect is much milder and it cannot explain the partial crossing. The corotating and timeaccurate simulations also show relatively mild bending of the current sheet. The difference between the numerical results may be attributed to several factors: we used Uranus data as opposed to scaled Earth simulations and the numerical schemes are also different. In our opinion the partial crossing can be best explained with dynamic features in the magnetotail which are caused by temporal changes in the solar wind. Considering the limited data and the simplifications made in the model, the overall agreement of the simulated and observed data is remarkable.

\subsection{Time-Dependent Simulations}

[40] Our time-dependent simulation is started from a corotating steady state solution with the same physical parameters as the second corotating simulation discussed in the previous section, i.e., the solar wind density is taken to be $0.1 \mathrm{amu} / \mathrm{cm}^{3}$. In order for the simulation to run in a reasonable time, the finest cells near Uranus had to be coarsened from $1 / 8 R_{U}$ to $1 / 4 R_{U}$. In order to use the steady state corotating solution as an initial condition, the solution has to be transformed into the USO frame. We pick an initial time for the simulation when the USO and USM frames coincide, so only the $Y$ and $Z$ components of the velocity have to be transformed with the $\mathbf{u}_{U S O}=\mathbf{u}_{U S M}+$ $\boldsymbol{\Omega} \times \mathbf{d}$ equation.

[41] In the time-dependent simulation, Uranus is rotating around its real rotation axis, which is slightly tilted relative to the $X_{U S O}$ axis, thus slightly breaking cylindrical symmetry. The initial tilt of the magnetic dipole is $39.29^{\circ}$ in the $X-$ $Z$ plane of the USO system. The magnetic dipole is offset along the tilted rotation axis, thus initially the dipole is at $x$ $=-0.307 \mathrm{R}_{\mathrm{U}}$ and $z=0.043 \mathrm{R}_{\mathrm{U}}$.

[42] The block adaptive grid, although as symmetric as possible, also breaks the cylindrical symmetry due to discretization errors. The grid consists of 5120 blocks, or around 320,000 cells. To eliminate the effect of the initial transients due to the differences in the geometry and the discretization, we let the model run for 2 Uranian days in the USO frame in time-accurate mode. Then we extract the data along Voyager's path during a 4.5 rotation period that corresponds to the part of the trajectory lying between $50 \mathrm{R}_{\mathrm{U}}$ and $-100 \mathrm{R}_{\mathrm{U}}$ in the $X_{U S O}$ coordinate.

[43] We use the explicit-implicit time-stepping scheme with a fixed $75 \mathrm{~s}$ time step, which gives the best computational speed for this simulation. About half of the 5120 blocks use implicit time stepping, while for the other half the stability conditions permit explicit time stepping. Although this scheme is almost 90 times faster than the explicit time stepping, the simulation of the 6.5 Uranian days (roughly 4.6 Earth days) requires almost a full day on 30 processors of our PC cluster.

[44] The comparison of simulated and observed magnetic data in the magnetotail is shown in Figure 12. The agreement is rather good, although the RMS deviation is somewhat more than in the second corotating simulation in Figure 8 . The difference seems to be caused by a small shift in the steep gradient of the $B_{x}$ component near $x=$ -35 . It is difficult to tell whether this shift is due to the 

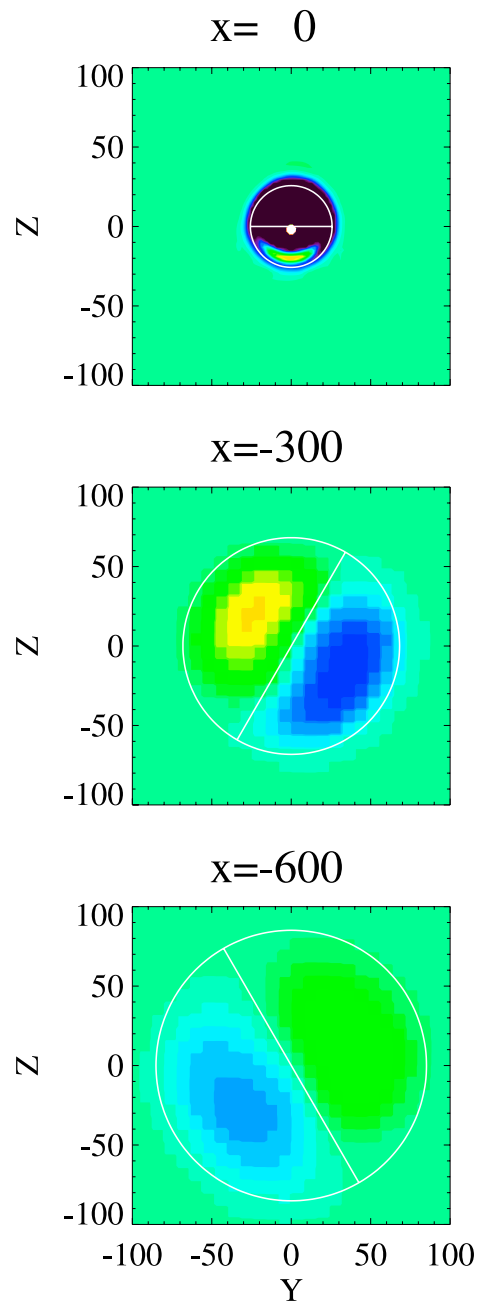

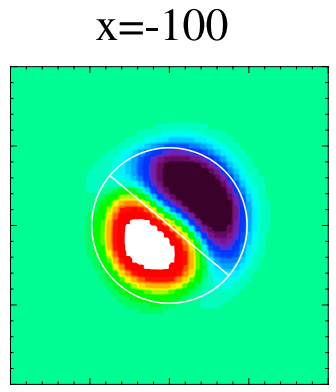

$x=-400$
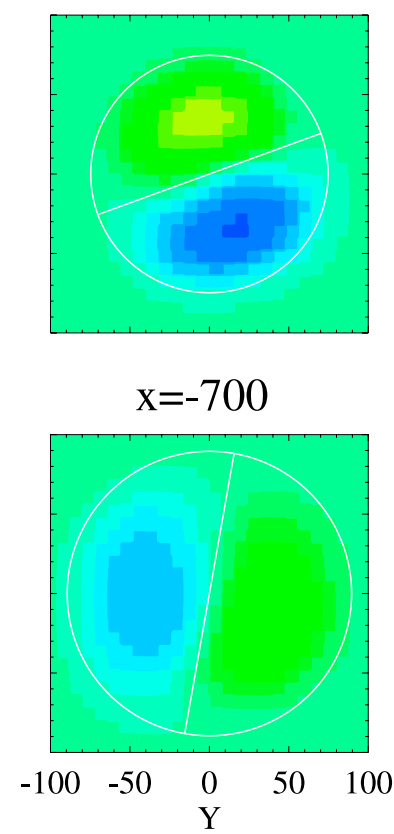

$x=-200$

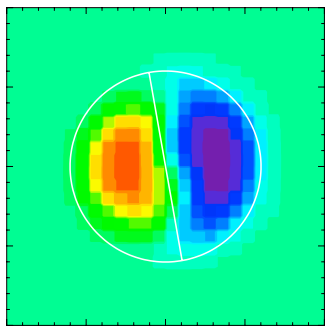

$x=-500$
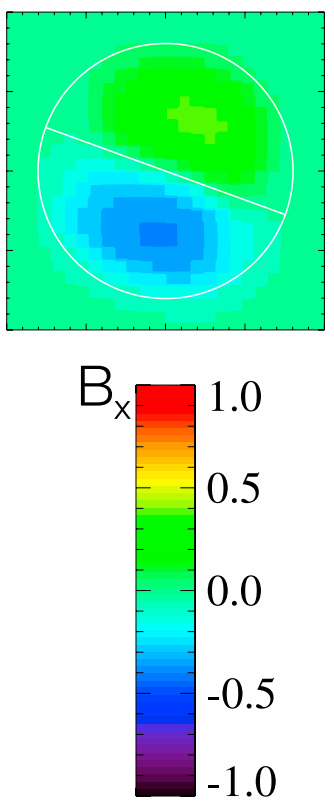

Figure 17. The $B_{x}$ component of the magnetic field at various cuts orthogonal to the $X$ axis in the second $\left(\rho_{S W}=0.1 \mathrm{amu} / \mathrm{cm}^{3}\right)$ corotating simulation. The color scale is saturated in the $x=0$ and $x=$ -100 cuts. The circles correspond to the cubic fit in equation (10), while the tilted diameters show the $\alpha=0.4^{\circ} x / \mathrm{R}_{\mathrm{U}}$ fit to the orientation of the current sheet.

coarser resolution, the discretization errors caused by the rotation, insufficient time for the time-accurate simulation to relax, or some other reason. Overall, however, the time-accurate simulation agrees remarkably well with the observations.

[45] We also investigated how much the loss of the cylindrical symmetry affects the solution. The cylindrical symmetry is broken by the tilt of the rotation axis as well as by the discretization errors due to the Cartesian grid blocks. Figure 13 shows the variation of the $x$ component of the magnetic field. We chose this quantity because it nicely depicts the structure of the magnetotail. The eight panels in the figure contain the simulation results separated by one quarter of a Uranian day. Comparing every second plot shows that in half a day the solution flips by $180^{\circ}$. The tilt of the rotation axis does not have any obvious effect, since the solar wind, which blows exactly from the $x$ direction, dominates in the magnetotail. A more careful inspection of the figure, however, reveals that the contour lines hitting the left edge of the plots at $x=-500 \mathrm{R}_{\mathrm{U}}$ are not perfectly symmetric when the panels at $t=3.25$ and 4.25 Uranian days are compared with the panels $t=3.75$ and 4.75 Uranian days. This small asymmetry may be due to the tilt of the rotation axis. Comparison of the left and right columns in Figure 13 demonstrates that the solution is almost exactly periodic. We find that the time-accurate solution can be regarded as a steady state solution in the corotating frame with a very good approximation.

[46] The time-dependent shape of the bow shock is depicted in Figure 14. At 3.00 Uranian day after the beginning of the simulation the dipole is pointing toward $+Z$ with a $39^{\circ}$ tilt toward the Sun. At 3.25 days the dipole points toward the $-Y$ axis (out of the plane of the figure) with a $31^{\circ}$ tilt. At 3.50 days the dipole points toward $-Z$ with a $23^{\circ}$ tilt toward the Sun. Finally, at 3.75 days it points toward the $+Y$ axis (into the plane of the figure) with a $31^{\circ}$ tilt. The overall shape of the bow shock is well fitted with

$$
x=22 \mathrm{R}_{\mathrm{U}}-\frac{d^{2}}{65 \mathrm{R}_{\mathrm{U}}},
$$

where $x$ and $d=\sqrt{y^{2}+z^{2}}$ are both measured in units of the planet radius. Close to the body, the bow shock is bulging 


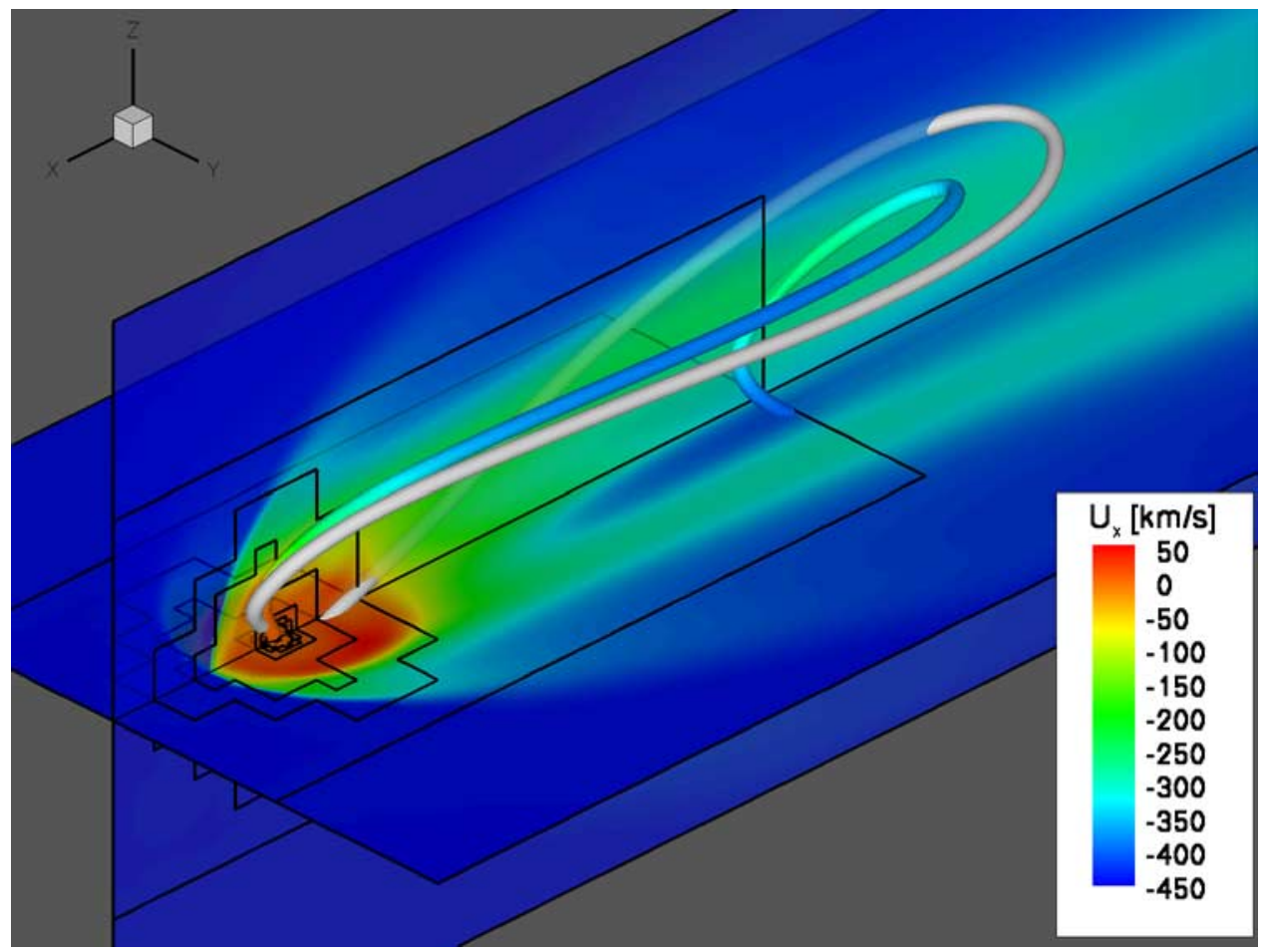

Figure 18. The $u_{x}$ velocity space and two magnetic field lines in the corotating frame. The velocity component values are shown on $X-Y$ and $X-Z$ planes where black lines separate regions of different grid resolutions. The white field line is closed, crosses the $X-Z$ plane once further and twice closer to the planet. The other field line that is colored by velocity also turns back and intersects the $X-Y$ plane at the outmost resolution change. Note how the color (the velocity) of the field line is correlated with its shape and twists.

out at the magnetic pole pointing toward the Sun. Owing to the rotation of the planet, the bulge caused by the tilt of the dipole propagates downtail in a spiraling motion, and this makes the snapshots at 3.25 days and 3.75 days asymmetric, although at these times the dipole is in the $Z=0$ plane. The bulge can be approximately fitted with the

$$
x=22 \mathrm{R}_{\mathrm{U}}-\frac{d^{2}}{80 \mathrm{R}_{\mathrm{U}}}
$$

half parabola for $x>-50 \mathrm{R}_{\mathrm{U}}$ at all four times shown. The symmetry of the plots at 3.0 and 3.5 Uranian days suggest that the $8^{\circ}$ tilt of the rotation axis relative to the $X$ axis has relatively little effect on the shape of the bow shock.

\section{Conclusions}

[47] On the basis of the comparison with the Voyager data we believe that we have successfully reconstructed the 3-D magnetosphere of Uranus during the flyby. The magnetic data is well approximated with a steady state solution in the corotating USM frame. The asymmetric shape of the bow shock due to the large tilt angle of the magnetic dipole is partially captured even by the much simplified inertial steady state solution (see Figure 6), but the correct shape can only be obtained with a corotating steady state or a fully time-accurate simulation (see Figure 14).

[48] The open field lines follow a helical pattern as shown in Figure 15 and expected from physical intuition.
The twisted structure of the magnetotail is visualized in Figure 16 with an isosurface at $|B|=0.5 \mathrm{nT}$. On the basis of cuts orthogonal to the $X$ axis (as shown in Figure 17), the current sheet is twisted by about $h=0.4^{\circ} / \mathrm{R}_{\mathrm{U}}$ at a roughly constant rate. Combined with the rotation period of Uranus, this twisting rate corresponds to a propagation speed $v=\Omega / h \approx 370 \mathrm{~km} / \mathrm{s}$. The twisting rate is determined by a balance between the magnetic stress, which propagates with the Alfvén speed, and the friction against the surrounding solar wind which is not rotating together with the planet. The Alfvén wave speed $v_{A}=\left|u_{x}\right|+\left|B_{x}\right| / \sqrt{\mu_{0} \rho}$ is between $450 \mathrm{~km} / \mathrm{s}$ and $600 \mathrm{~km} / \mathrm{s}$ in most of the magnetotail, which is somewhat larger than the propagation speed of the twist, as expected. To put it in another way, if there was no friction, the magnetotail would be twisted by $h_{A}=\Omega / v_{A} \approx$ $0.28^{\circ} / \mathrm{R}_{\mathrm{U}}$, but due to the friction against the solar wind, the lobes are more twisted with $h=0.4^{\circ} / \mathrm{R}_{\mathrm{U}}$. The extra twist provides the magnetic stress which balances the friction forces.

[49] If the threshold of the isosurface is lowered to $|B|=$ $0.01 \mathrm{nT}$ or below, the whole magnetotail is captured. The cross section with the $z_{U S M}=0$ plain is reasonably approximated with the cubic paraboloid

$$
x=16 \mathrm{R}_{\mathrm{U}}-\frac{d^{3}}{1000 \mathrm{R}_{\mathrm{U}}^{2}}
$$

for $x>-300 \mathrm{R}_{\mathrm{U}}$. The cross section with the $y_{U S M}=0$ plain is rather asymmetric and cannot be well fitted with a simple curve. 
[50] The visualization of a closed field line is shown in Figure 18. The field line is stretched toward the tail and twisted due to the rotation. What is less intuitive is that the stretch of the field lines is not homogeneous. As the field line passes through regions with varying plasma speeds, it is stretched by different amounts. This may cause the $B_{x}$ component to become zero even for the open field lines, as shown in the figure. Owing to this nonuniform stretching some of the open field lines projected onto the $Y=0$ or $Z=$ 0 planes may appear as having loops.

[51] Our results indicate that the MHD equations solved with efficient parallel numerical algorithms are capable of reproducing the basic features of the three-dimensional magnetosphere of Uranus from a single 1-D data set. The quantitative comparison of the simulated and observed data suggests that the solar wind density was around $0.1 \mathrm{amu} / \mathrm{cm}^{3}$ while Voyager was inside the magnetosphere. The simulations presented in this paper reveal the dynamics of a strongly tilted rotating dipole interacting with the solar wind. In the future we plan to model Neptune, which also has a strongly tilted dipole, and the rotation axis is not aligned with the solar wind. We expect therefore a fully time-dependent solution, which cannot be described as a steady state in any frame. On the basis of our experience with the Uranus simulations we are hopeful that we will be able to model the even more challenging magnetosphere of Neptune as well.

[52] Acknowledgments. We are grateful to Steve Joy and Joe Mafi at the Planetary Plasma Interactions (PPI) Node of NASA's Planetary Data System (PDS) for providing us with the Voyager data. G.T. and D.K. have been partly supported by the the Hungarian Science Foundation (OTKA, grants T037548 and T047042) and by the Education Ministry of Hungary (grant FKFP-0242-2000). At the University of Michigan this work has been supported by NASA IASRP grant NAG5-9406, NASA ESS CT cooperative agreement NCC5-614, and by NASA grant NAGW-1366.

[53] Lou-Chuang Lee thanks Stephen A. Ledvina and Raymond J. Walker for their assistance in evaluating this paper.

\section{References}

Behannon, K. W., R. P. Lepping, E. C. Sittler Jr., N. F. Ness, and B. H. Mauk (1987), The magnetotail of Uranus, J. Geophys. Res., 92, 15,354.
Bridge, H. S., J. W. Belcher, B. Coppi, A. J. Lazarus, R. L. McNutt Jr. S. Olbert, R. E. Hartle, K. W. Ogilvie, E. C. Sittler Jr., and R. S. Wolfe (1986), Plasma observations near Uranus: Initial results from Voyager 2, Science, 233, 89.

Connerney, J. E. P., H. M. Acuña, and N. F. Ness (1987), The magnetic field of Uranus, J. Geophys. Res., 92, 15,329.

Hammond, C. M., R. J. Walker, and M. G. Kivelson (1990), A pincershaped plasma sheet at Uranus, J. Geophys. Res., 95, 14,987.

Harten, A. (1983), High resolution schemes for hyperbolic conservation laws, J. Comput. Phys., 49, 357.

Keppens, R., G. Tóth, M. A. Botchev, and A. van der Ploeg (1999), Implicit and semi-implicit schemes: Algorithms, Int. J. Numer. Methods Fluids, 30,335 .

Lepping, R. P. (1994), Comparisons of the field configurations of the magnetotails of Uranus and Neptune, Planet. Space. Sci., 42, 847.

Ness, N. F., H. M. Acuña, K. W. Behannon, L. F. Burlaga, J. E. P. Connerney, and R. P. Lepping (1986), Magnetic fields at Uranus, Science, 233,85 .

Ogino, T., and R. J. Walker (1984), A magnetohydrodynamic simulation of the bifurcation of tail lobes during intervals with a northward interplanetary magnetic field, Geophys. Res. Lett., 11, 1018-1021.

Powell, K. G. (1994), An approximate Riemann solver for magnetohydrodynamics (that works in more than one dimension), Rep. 94-24, Inst. for Comput. Appl. in Sci. and Eng., Langley, Va.

Powell, K. G., P. L. Roe, T. J. Linde, T. I. Gombosi, and D. L. De Zeeuw (1999), A solution-adaptive upwind scheme for ideal magnetohydrodynamics, J. Comput. Phys., 154(2), 284-309, doi:10.1006/ jcph.1999.6299.

Sokolov, I. V., E. V. Timofeev, J.-I. Sakai, and K. Takayama (2002), Artificial wind-A new framework to construct simple and efficient upwind shock-capturing schemes, J. Comput. Phys., 181(1), 354-393, doi:10.1006/jcph.2002.7130.

Tanaka, T. (1994), Finite volume TVD scheme on an unstructured grid system for three-diemsnional MHD simulations of inhomogeneous systems including strong background potential field, J. Comput. Phys., 111, 381-389, doi:10.1006/jcph.1994.1071.

Tóth, G., R. Keppens, and M. A. Botchev (1998), Implicit and semi-implicit schemes in the Versatile Advection Code: Numerical tests, Astron. Astrophys., 332, 1159.

van Leer, B. (1979), Towards the ultimate conservative difference scheme. V. A second order sequel to Godunov's method, J. Comput. Phys., 32, 101.

T. I. Gombosi, K. C. Hansen, and G. Tóth, Space Physics Research Laboratory, Department of Atmospheric, Oceanic and Space Sciences, University of Michigan, Ann Arbor, MI 48109-2143, USA. (gtoth@ umich.edu)

D. Kovács, Department of Atomic Physics, Eötvös University, Pázmány sétány 1/A, Budapest H-1117, Hungary. 